\title{
On neutrinoless double beta decay in the minimal left-right symmetric model
}

\author{
Wei-Chih Huang ${ }^{1,2,3, a}$, J. Lopez-Pavon ${ }^{1,2, b}$ \\ ${ }^{1}$ SISSA, via Bonomea 265, 34136 Trieste, Italy \\ ${ }^{2}$ INFN, sezione di Trieste, 34136 Trieste, Italy \\ ${ }^{3}$ Department of Physics and Astronomy, University College London, London WC1E 6BT, UK
}

Received: 31 January 2014 / Accepted: 9 April 2014 / Published online: 6 May 2014

(C) The Author(s) 2014. This article is published with open access at Springerlink.com

\begin{abstract}
We analyze the general phenomenology of neutrinoless double beta decay in the minimal left-right symmetric model. We study under which conditions a New Physics dominated neutrinoless double beta decay signal can be expected in the future experiments. We show that the correlation among the different contributions to the process, which arises from the neutrino mass generation mechanism, can play a crucial role. We have found that, if no fine tuned cancelation is involved in the light-active neutrino contribution, a New Physics signal can be expected mainly from the $W_{R}-W_{R}$ channel. An interesting exception is the $W_{L^{-}}$ $W_{R}$ channel which can give a dominant contribution to the process if the right-handed neutrino spectrum is hierarchical with $M_{1} \lesssim \mathrm{MeV}$ and $M_{2}, M_{3} \gtrsim \mathrm{GeV}$. We also discuss if a New Physics signal in neutrinoless double beta decay experiments is compatible with the existence of a successful Dark Matter candidate in the left-right symmetric models. It turns out that, although it is not a generic feature of the theory, it is still possible to accommodate such a signal with a $\mathrm{KeV}$ sterile neutrino as dark matter.
\end{abstract}

\section{Introduction}

The recent LHC results [1,2] seem to indicate that the Higgs mechanism, with the Higgs mass around $125 \mathrm{GeV}$, is the responsible for the mass generation of the Standard Model (SM) particles. However, the origin of light neutrino masses, for the existence of which we have compelling evidence from neutrino oscillation experiments, still remains unknown. It is true that the light neutrino masses could also be generated through the Higgs mechanism in a minimally extended SM which includes sterile (right-handed) neutrino fields as

\footnotetext{
a e-mail: wei-chih.huang@ucl.ac.uk

b e-mail: jlpavon@sissa.it
}

$S U(2)_{L}$ singlets and in which the total lepton number is conserved. However, their smallness in comparison with the charged lepton and quark masses calls for a different explanation. In this context, extensions of the SM required to explain the origin of neutrino masses, and compatible with the latest LHC data, arise as quite suggestive models of New Physics (NP). Among those we find the celebrated seesaw models [3-6], which can give, in addition, the key to explain the matter-antimatter asymmetry of the universe through leptogenesis [7].

Most of those models predict that neutrinos are Majorana particles, something which can be tested in lepton number violating processes such as the neutrinoless double beta $(0 \nu \beta \beta)$ decay. The $0 \nu \beta \beta$ decay experiments are the most promising ones in this context but they suffer a serious drawback: the NP contribution to the process is usually short range and thus typically very suppressed compared to that of the light neutrinos. Thanks to the future $0 \nu \beta \beta$ experiments [8-14], in combination with the complementary information coming from neutrino oscillation experiments and cosmology, we might be able to discover the Majorana nature of neutrinos, but not easily which is the mechanism responsible for the neutrino mass generation $[15,16]$. In this context, the correlations between the standard light neutrino and NP contribution to the $0 \nu \beta \beta$ decay are crucial, as shown in the case of the type-I [3-6], type-II [17-21] and type-III [22] seesaw models in Refs. [23,24]. The generation of light neutrino masses in a particular model usually induces important correlations between the different contributions to the $0 \nu \beta \beta$ decay, which should always be considered in a model dependent analysis, helping to understand which type of NP it is feasible to test in the experiments.

In this work we will focus on the $0 \nu \beta \beta$ decay phenomenology of the minimal left-right symmetric model (MLRSM) [6,21,25-27]. The left-right symmetric models have been widely studied in the literature since, among other features, 
they provide a natural explanation for the smallness of the neutrino masses (some recent analysis in the context of the $0 \nu \beta \beta$ decay can be found in Refs. [28-35]). In our analysis we will assume that no accidental cancelation occurs in the light neutrino mediated $W_{L}-W_{L}$ channel, which involves the exchange of two $W_{L}$. We will distinguish three regions of the parameter space depending on the mass of the righthanded $(\mathrm{RH})$ neutrinos. First, we will show that if the righthanded $(\mathrm{RH})$ neutrinos are heavier than the $0 \nu \beta \beta$ decay scale $(\sim 100 \mathrm{MeV})$, the $0 \nu \beta \beta$ decay rate is dominated by light neutrino exchange channels with the exception of the channel in which two $W_{R}$ are exchanged $\left(W_{R}-W_{R}\right.$ channel) mediated by heavy neutrinos ${ }^{1}$. One of the light neutrino mediated channels involves the exchange of one $W_{L}$ and one $W_{R}\left(W_{L}-W_{R}\right.$ channel); however, it turns out that a NP dominant contribution can come mainly from the $W_{R}-W_{R}$ channel. Secondly, we will study the region of the parameter space where the $\mathrm{RH}$ neutrinos are lighter than the $0 v \beta \beta$ scale. We have found that in this case the $W_{L}-W_{R}$ contribution cancels out while a NP signal can still be expected from the $W_{R}-W_{R}$ channel. In this region, the $\mathrm{RH}$ neutrinos can give a relevant contribution through the $W_{L}-W_{L}$ channel, as opposed to the type-I seesaw case where the total $W_{L}-W_{L}$ contribution is very suppressed. Finally, we will investigate a mixed scenario with RH neutrinos in both regions below and above the $0 \nu \beta \beta$ decay scale. We have found that this is the only scenario in which the $W_{L^{-}}$ $W_{R}$ channel turns out to be relevant and can be responsible of a future signal (if no cancelation in the $W_{L}-W_{L}$ channel is invoked). In all the cases we will show for which part of the parameter space a NP signal in future $0 \nu \beta \beta$ decay experiments can be expected. Moreover, we will also analyze if such a signal can be compatible with the existence of a successful Dark Matter (DM) candidate in the left-right symmetric model, study the complementary bounds coming from charged lepton flavor violation (LFV) experiments and the impact of the one-loop corrections to the light neutrino masses.

This work is organized as follows. In Sect. 2 we briefly describe the MLRSM, focusing on the relations among the parameters of the model induced by the neutrino mass generation. In Sect. 3 we analyze the neutrinoless double beta decay phenomenology in the MLRSM, studying in particular for which part of the parameter space a $0 \nu \beta \beta$ decay signal coming mainly from NP contributions can be possible. Section 4 is devoted to the analysis of complementary constraints coming mainly from charged LFV experiments and the stability of the light neutrino masses under one-loop corrections. In Sect. 5 we study if a successful DM candidate and a NP signal in the future $0 \nu \beta \beta$ decay experiments can be compatible in the MLRSM. Finally, we conclude in Sect. 6.

${ }^{1}$ If other contributions coming from different models are not involved.

\section{Minimal left-right symmetric model and neutrino masses}

The Lagrangian of the MLRSM respects an enlarged gauge symmetry $S U(3)_{c} \otimes S U(2)_{L} \otimes S U(2)_{R} \otimes U(1)_{B-L}$ plus a discrete left-right symmetry which leads to equal $S U(2)_{L}$ and $S U(2)_{R}$ gauge couplings $\left(g_{L}=g_{R}=g\right)$. We are not going into the details of the model since it has been widely studied in the literature (for a recent complete analysis regarding the associated lepton number violating effects, see for instance Refs. [29,33]), but only recall the most relevant features for our analysis. The scalar sector is also augmented by the addition of two scalar triplets $\left(\Delta_{L}\right.$ and $\left.\Delta_{R}\right)$ and a bi-doublet scalar under $S U(2)_{L} \otimes S U(2)_{R}$, which spontaneously break the electroweak symmetry when they develop vacuum expectation values (VEVs).

In this section we will derive the relations which will be used in the phenomenological analysis of the $0 v \beta \beta$ decay. Since they come from the neutrino mass generation, let us recall how the complete neutrino mass matrix looks like after the electroweak symmetry breaking:

$M_{\nu}=\left(\begin{array}{ll}M_{L} & m_{D}^{T} \\ m_{D} & M_{R}\end{array}\right)=U \operatorname{Diag}(m, M) U^{T}$,

where $m_{i}$ are the light neutrino masses and $M_{i}$ the heavy ones. Notice that in this model the Majorana mass term for the heavy neutrinos is generated dynamically when $\Delta_{R}$ takes a VEV $\left(M_{R}=Y_{\Delta_{R}} v_{R}\right)$, while the Majorana mass term $M_{L}$ for the left-handed (LH) neutrinos is generated analogously through the $\Delta_{L} \operatorname{VEV}\left(M_{L}=Y_{\Delta_{L}} v_{L}\right)$. The neutrino mass matrix is diagonalized as shown above by a $6 \times 6$ unitary matrix $U$, through the following rotation between the neutrino flavor and mass eigenstates denoted by $\alpha, \beta=e, \mu, \tau$ and $i, k=1,2,3$, respectively:

$$
\left(\begin{array}{c}
v_{\alpha L} \\
N_{\beta R}^{c}
\end{array}\right)=U\left(\begin{array}{c}
v_{i} \\
N_{k}
\end{array}\right)=\left(\begin{array}{cc}
\tilde{U} & B \\
A & V
\end{array}\right)\left(\begin{array}{c}
v_{i} \\
N_{k}
\end{array}\right) \text {. }
$$

The diagonalization of the complete neutrino mass matrix presented in Eq. (1) provides the following useful relations:

$\tilde{U} m \tilde{U}^{T}+B M B^{T}=M_{L}$,

$\tilde{U} m A^{T}+B M V^{T}=m_{D}^{T}$,

$A m A^{T}+V M V^{T}=M_{R}$.

On the other hand, taking into account that the active $\mathrm{LH}$ block of $U, \tilde{U}$, is unitary to a very good approximation (at least up to the percent level [36]), the complete neutrino mixing matrix can be expanded as

$$
\begin{aligned}
U & =\left(\begin{array}{cc}
1-\theta \theta^{\dagger} / 2 & \theta \\
-\theta^{\dagger} & 1-\theta^{\dagger} \theta / 2
\end{array}\right)\left(\begin{array}{cc}
U_{\mathrm{pmns}} & 0 \\
0 & V
\end{array}\right)+\mathcal{O}\left(\theta^{3}\right) \\
& =\left(\begin{array}{cc}
U_{\mathrm{pmns}} & \theta V \\
-\theta^{\dagger} U_{\mathrm{pmns}} & V
\end{array}\right)+\mathcal{O}\left(\theta^{2}\right)
\end{aligned}
$$


where $\theta$ is a $3 \times 3$ matrix which characterizes the small mixing between the active $\mathrm{LH}$ and the heavy $\mathrm{RH}$ neutrinos, $U_{\mathrm{pmns}}$ is the PMNS matrix and $V$ is a $3 \times 3$ unitary matrix. From Eqs. (3-5), we have

$U_{\mathrm{pmns}} m U_{\mathrm{pmns}}^{T}=M_{L}-\theta M_{R} \theta^{T}$,

$\theta M_{R}-M_{L} \theta^{*}=m_{D}^{T}$

$V M V^{T}=M_{R}\left(1+\mathcal{O}\left(\frac{M_{L}}{M_{R}} \theta^{2}\right)\right)$.

The discrete (charge conjugation) LR symmetry gives the following relation between the Yukawa couplings of the triplets: $Y_{\Delta_{R}}=Y_{\Delta_{L}} \equiv Y_{\Delta} \cdot{ }^{2}$ This means that

$\left(M_{L}\right)_{\alpha \beta} /\left(M_{R}\right)_{\alpha \beta}=v_{L} / v_{R}<10^{-3}$,

where we have employed the present bounds on $v_{L}$ and $v_{R}$, namely $v_{L} \lesssim 7 \mathrm{GeV}$ [37] and $v_{R} \gtrsim 10 \mathrm{TeV}\left(M_{W_{R}} \approx\right.$ $g v_{R} / \sqrt{2} \gtrsim \mathrm{TeV}$ [38-40]). Therefore, the $\mathcal{O}\left(M_{L} \theta^{*}\right)$ and $\mathcal{O}\left(\frac{M_{L}}{M_{R}} \theta^{2}\right)$ can be safely neglected in Eq. (8) and Eq. (9), respectively, and

$\theta \simeq m_{D}^{T} M_{R}^{-1}$

Of course, $\theta$ plays a fundamental role at the phenomenological level since it basically describes the mixing between the active $\mathrm{LH}$ neutrinos and the $\mathrm{RH}$ ones. It would be very interesting thus to find a useful parameterization of $\theta$ as a function of the light neutrino parameters, light neutrino masses and the angles/phases of the PMNS matrix, and the rest of the independent parameters of the model associated with the RH neutrino sector. In principle, an analogous parameterization to the Casas-Ibarra one [41] would be a good candidate [42]. However, the presence of $M_{L}$ in Eq. (7) and the fact that the matrix $V$ is in this case physical, contrary to the type-I seesaw model, makes that parameterization less transparent and more involved than expected. On the other hand, the discrete (charge conjugation) LR symmetry leads to the following constraint:

$m_{D}=m_{D}^{T}$,

and thus Eq. (11) becomes

$\theta M_{R}=M_{R} \theta^{T}=m_{D}$.

Plugging this relation and Eq. (9) into Eq. (7), we obtain,

$U_{\mathrm{pmns}} m U_{\mathrm{pmns}}^{T}=M_{L}-\theta^{2} V M V^{T}$,

and finally with $Y_{\Delta_{R}}=Y_{\Delta_{L}} \equiv Y_{\Delta}$ and hence $M_{L}=\frac{v_{L}}{v_{R}} M_{R}$, we have

$\theta=\left[\frac{v_{L}}{v_{R}} I-U_{\mathrm{pmns}} m U_{\mathrm{pmns}}^{T} V^{*} M^{-1} V^{\dagger}\right]^{1 / 2}$.

2 Another option is to consider instead a discrete parity symmetry leading to a similar relation: $Y_{\Delta_{R}}=Y_{\Delta_{L}}^{*} \equiv Y_{\Delta}$.
Therefore, $\theta$ is completely determined as a function of the light and heavy neutrino masses, $m$ and $M$, the PMNS matrix, $U_{\text {pmns }}, v_{L} / v_{R}$, and the unitary matrix $V$ [31]. Notice that if this expression is used to obtain $\theta$ with the PMNS mixing angles and the solar and atmospheric mass-squared differences as input parameters, we ensure that the model is consistent with the light neutrino mass and mixing pattern measured in neutrino oscillation experiments.

\section{Neutrinoless double beta decay}

In our study of the $0 \nu \beta \beta$ decay in the MLRSM, we will pay special attention to the correlation among all the contributions to the process and, in particular, the connection with the light neutrino masses. We shall see that the correlation between the different contributions and the experimental bounds on the parameters will allow us to safely neglect some of the NP contributions.

As we have already mentioned, we will not analyze the scenario in which a cancelation occurs within the standard light neutrino contribution, which would naively leave the NP channels as the leading contributions $[43,44]$. Of course, this cancelation can be due to the presence of an extra symmetry added to the model, such as the lepton number which is approximately conserved in the so called inverse or direct seesaw models [45-49]. The problem in this scenario is that, in order for the NP contributions to be measurable, a significant violation of lepton number should be introduced through the NP sector which may not have an impact on the light neutrino masses at tree level but arises naturally at one-loop level, as shown in Ref. [23] in the context of the seesaw models. This makes it very difficult to have a significant contribution from NP channels since the one-loop correction to the light neutrino masses tends to dominate in the $0 v \beta \beta$ decay rate.

We will distinguish three different regions according to the associated $0 \nu \beta \beta$ decay phenomenology: (i) when the $\mathrm{RH}$ neutrinos are much heavier than the $0 v \beta \beta$ decay scale $(\langle p\rangle \approx$ $100 \mathrm{MeV})$, which means heavier than approximately $1 \mathrm{GeV}$; (ii) when the $\mathrm{RH}$ neutrinos are much lighter than the $0 \nu \beta \beta$ decay scale (below $1 \mathrm{MeV}$ ); (iii) when the RH neutrinos are in both regions, (i) and (ii).

In the analysis below we have reasonably estimated the NMEs corresponding to some of the channels under study. This is accurate enough for our purposes but, although the associated NMEs errors are still large, in order to be more precise, the full calculation of all the NMEs should be considered.

\subsection{Heavy regime}

The various contributions to the $0 \nu \beta \beta$ transition rate in this model are described by the Feynman diagrams shown in 
Fig. 1 Feynman diagrams contributing to the $0 \nu \beta \beta$ transition rate in the MLRSM

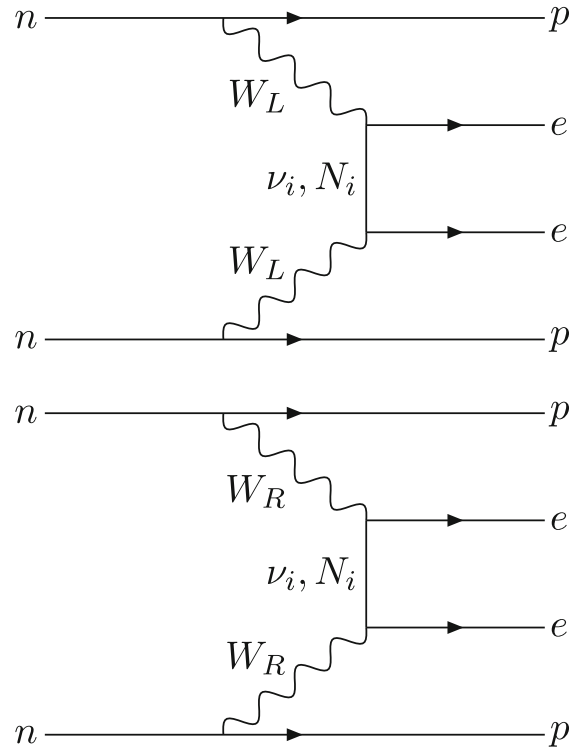

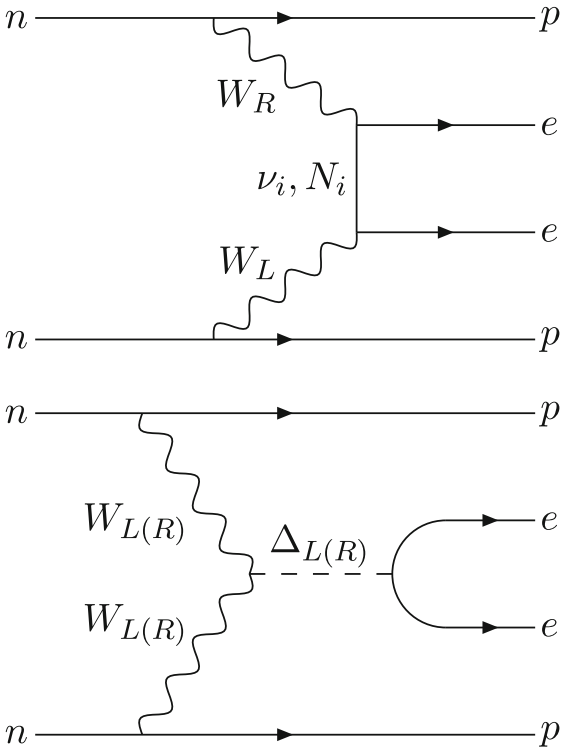

Fig. 1. We will start describing them one by one in order to show that the contributions of the diagrams in which a heavy fermion (or scalar) is exchanged are subdominant with respect to those of the light neutrino exchange, the only exception being the $W_{R}-W_{R}$ channel.

- $\mathbf{W}_{\mathbf{L}}-\mathbf{W}_{\mathbf{L}}$ channel. The amplitude corresponding to the top left diagram of Fig. 1 is given by

$$
\begin{aligned}
& A_{L L} \propto(1+\mathcal{O}(\xi))\left[\sum_{i=1}^{3} m_{i} \tilde{U}_{e i}^{2} \mathcal{M}^{0 \nu \beta \beta}(0)\right. \\
& \left.+\sum_{i=1}^{3} M_{i} B_{e i}^{2} \mathcal{M}^{0 \nu \beta \beta}\left(M_{i}\right)\right]
\end{aligned}
$$

where $\xi$ is the $W_{L}-W_{R}$ mixing angle. The present experimental bound is given by $\xi \lesssim 10^{-2}$ [38], however, in the minimal left-right symmetric model there is a stronger theoretical upper bound given by $M_{W_{L}}^{2} / M_{W_{R}}^{2}<$ $10^{-3}$ [40]. ${ }^{3}$ In the case of the $W_{L}-W_{L}$ contribution, $\xi$ can be safely neglected. $\mathcal{M}^{0 \nu \beta \beta}$ are the associated nuclear matrix elements (NMEs) following the notation of Ref. [24], where the NMEs were computed as a function of the mass of the neutrino mediating the process for different nuclei. Notice that in this notation the NMEs include the dependence on the propagator. The NMEs corresponding to the light neutrino exchange are independent of the neutrino masses, and then with Eq. (3), the above amplitude can be rewritten as

\footnotetext{
${ }^{3}$ Notice that $\xi$ can only saturate this bound if and only if the two VEVs of the Higgs doublets are of the same order.
}

$$
\begin{aligned}
& A_{L L} \propto\left(M_{L}\right)_{e e} \mathcal{M}^{0 \nu \beta \beta}(0) \\
& +\sum_{i=1}^{3} M_{i} B_{e i}^{2}\left(\mathcal{M}^{0 \nu \beta \beta}\left(M_{i}\right)-\mathcal{M}^{0 \nu \beta \beta}(0)\right) .
\end{aligned}
$$

Taking into account that $\mathcal{M}^{0 \nu \beta \beta}\left(M_{i}\right) / \mathcal{M}^{0 \nu \beta \beta}(0) \ll 1$ [24], the contribution due to the heavy neutrino exchange can be safely neglected. Using again Eq. (3), one obtains,

$$
\begin{aligned}
& A_{L L} \propto \sum_{i=1}^{3} m_{i} \tilde{U}_{e i}^{2} \mathcal{M}^{0 \nu \beta \beta}(0) \\
& =\sum_{i=1}^{3}\left(U_{\mathrm{pmns}} m U_{\mathrm{pmns}}^{T}\right)_{e e} \mathcal{M}^{0 \nu \beta \beta}(0),
\end{aligned}
$$

which is the standard light neutrino contribution.

- $\mathbf{W}_{\mathbf{R}}-\mathbf{W}_{\mathbf{R}}$ channel. The amplitude of the bottom left diagram of Fig. 1, in which two $W_{R}$ are involved, is given by [50]

$$
\begin{aligned}
& A_{R R} \propto\left(\frac{M_{W_{L}}^{2}}{M_{W_{R}}^{2}}+\xi\right)^{2}\left[\sum_{i=1}^{3} m_{i} A_{e i}^{* 2} \mathcal{M}^{0 \nu \beta \beta}(0)\right. \\
& \left.+\sum_{i=1}^{3} M_{i} V_{e i}^{* 2} \mathcal{M}^{0 \nu \beta \beta}\left(M_{i}\right)\right]
\end{aligned}
$$

Using Eq. (6) in the above equation we obtain

$$
\begin{gathered}
A_{R R} \propto\left(\frac{M_{W_{L}}^{2}}{M_{W_{R}}^{2}}+\xi\right)^{2}\left[\sum_{i=1}^{3} M_{i} V_{e i}^{* 2} \mathcal{M}^{0 \nu \beta \beta}\left(M_{i}\right)\right. \\
\left.-\left(\theta^{T} U_{\mathrm{pmns}}^{*} m U_{\mathrm{pmns}}^{\dagger} \theta\right)_{e e} \mathcal{M}^{0 \nu \beta \beta}(0)\right] .
\end{gathered}
$$


Clearly, the second term can be neglected in comparison with the standard contribution due to the double suppression coming from $\left(\frac{M_{W_{L}}^{2}}{M_{W_{R}}^{2}}+\xi\right)^{2}$ and the active-heavy mixing, at least $\left|\theta_{\alpha i}\right|^{2} \lesssim 10^{-2}[36,51,52]$. The first term, however, cannot be neglected, i.e.,

$$
A_{R R} \propto \sum_{i=1}^{3} M_{i} V_{e i}^{* 2}\left(\frac{M_{W_{L}}^{2}}{M_{W_{R}}^{2}}+\xi\right)^{2} \mathcal{M}^{0 v \beta \beta}\left(M_{i}\right)
$$

- $\mathbf{W}_{\mathbf{L}}-\mathbf{W}_{\mathbf{R}}$ channel. For the diagram in the top right of Fig. 1, in which $W_{L}$ and $W_{R}$ are exchanged, the amplitude is given by

$$
\begin{aligned}
& \left.A_{L R} \propto\left(\xi+\eta \frac{M_{W_{L}}^{2}}{M_{W_{R}}^{2}}\right)\langle p\rangle\right] \sum_{i=1}^{3} A_{e i} \tilde{U}_{e i}^{*} \mathcal{M}^{0 \nu \beta \beta}(0) \\
& \left.+\sum_{i=1}^{3} V_{e i} B_{e i}^{*} \mathcal{M}^{0 \nu \beta \beta}\left(M_{i}\right)\right],
\end{aligned}
$$

where $\eta \approx 10^{-2}[53-58]^{4}$. Taking into account that $U$ is unitary, we have

$$
\begin{aligned}
& A_{L R} \propto\left(\xi+\eta \frac{M_{W_{L}}^{2}}{M_{W_{R}}^{2}}\right)\langle p\rangle \\
& \quad \times \sum_{i=1}^{3} V_{e i} B_{e i}^{*}\left(\mathcal{M}^{0 \nu \beta \beta}\left(M_{i}\right)-\mathcal{M}^{0 \nu \beta \beta}(0)\right),
\end{aligned}
$$

and since $\mathcal{M}^{0 \nu \beta \beta}\left(M_{i}\right) / \mathcal{M}^{0 \nu \beta \beta}(0) \ll 1$, Eq. (21) becomes

$\left.A_{L R} \propto\left(\xi+\eta \frac{M_{W_{L}}^{2}}{M_{W_{R}}^{2}}\right)\langle p\rangle \sum_{i=1}^{3} A_{e i} \tilde{U}_{e i}^{*} \mathcal{M}^{0 \nu \beta \beta}(0)\right)$,

which implies that the light neutrino mediated contribution of the $W_{L}-W_{R}$ channel is again dominant over the heavy neutrino exchange.

- The amplitude corresponding to the scalar triplet $\Delta_{L}$ exchange (bottom right in Fig. 1 with $W_{L}$ and $\Delta_{L}$ ) is suppressed with the factor

$$
\frac{\left(M_{L}\right)_{e e}}{\sum_{i} \tilde{U}_{e i} m_{i}} \frac{\left\langle p^{2}\right\rangle}{M_{\Delta_{L}}^{2}}=\frac{\left(M_{L}\right)_{e e}}{\left(M_{L}-m_{D} M_{R}^{-1} m_{D}^{T}\right)_{e e}} \frac{\left\langle p^{2}\right\rangle}{M_{\Delta_{L}}^{2}},
$$

\footnotetext{
${ }^{4}$ Notice that the first and second terms in Eq. (20) correspond to the mechanisms usually called $\eta$ and $\lambda$ mechanisms, respectively.
}

with respect to the standard contribution given in Eq. (17). The suppression factor is at least $\left\langle p^{2}\right\rangle / M_{\Delta_{L}}^{2} \ll 1$ if no fine tuned cancelation between the two terms in the light neutrino contribution is invoked, i.e., the contribution of this channel is negligible. For the corresponding "righthanded" version of the diagram the situation is slightly different and the suppression factor now reads

$$
\frac{M_{W_{L}}^{4}}{M_{W_{R}}^{4}} \frac{\left(M_{R}\right)_{e e}}{\left(M_{L}-m_{D} M_{R}^{-1} m_{D}^{T}\right)_{e e}} \frac{\left\langle p^{2}\right\rangle}{M_{\Delta_{R}}^{2}} .
$$

It seems that for small enough values of $\left(M_{L}-\right.$ $\left.m_{D} M_{R}^{-1} m_{D}^{T}\right)_{e e}=\sum_{i}\left[\left(U_{\mathrm{pmns}}\right)_{e i}\right]^{2} m_{i}$, this contribution could be larger than the standard one. However, it is not very easy to achieve a measurable $\Delta_{R}$ contribution, at the reach of the sensitivity of the next-to-next of $0 \nu \beta \beta$ decay experiments $\left(m_{\beta \beta} \sim 10^{-2} \mathrm{eV}\right)$. Indeed, the corresponding amplitude is given by

$$
\begin{gathered}
A_{\Delta_{R}} \propto\left(M_{R}\right)_{e e} \mathcal{M}_{\Delta}^{0 \nu \beta \beta}\left(M_{\Delta_{R}}\right) \\
\approx \frac{M_{W_{L}}^{4}}{M_{W_{R}}^{4}} \frac{\left\langle p^{2}\right\rangle}{v_{R}} \frac{\left(Y_{\Delta}\right)_{e e}}{2 \rho} \mathcal{M}^{0 \nu \beta \beta}(0),
\end{gathered}
$$

where we have used $M_{\Delta_{R}}^{2} \approx 2 \rho v_{R}^{2}$ and $\mathcal{L} \supset \rho \operatorname{Tr}\left(\Delta_{R} \Delta_{R}^{\dagger}\right.$ $\left.\Delta_{R} \Delta_{R}^{\dagger}\right)+Y_{\Delta} \overline{L_{R}^{c}} \Delta_{R} L_{R} .{ }^{5}$ The only possibility of having a phenomenologically relevant contribution is to saturate the bounds on $v_{R}\left(M_{W_{L}}^{4} / M_{W_{R}}^{4}<10^{-6}\right.$ [40] and $M_{W_{R}} \approx$ $v_{R} g / \sqrt{2}$ ) having at the same time $Y_{\Delta} \gg \rho$, which is not very feasible since a small value of $\rho$ would render $\Delta_{R}$ too light, contradicting the experimental bound, $m_{\Delta_{R}}>$ $320 \mathrm{GeV}$ [59]. We will thus neglect this contribution.

We have shown that only the contributions coming from the light neutrino exchange can have a significant impact on the $0 \nu \beta \beta$ decay rate, with the exception of the channel mediated by two $W_{R}$ gauge bosons in which the heavy neutrino exchange dominates. In summary, the phenomenologically relevant contributions to the $0 \nu \beta \beta$ decay rate can be recast as

$$
\begin{aligned}
& A_{\text {total }} \propto\left[c_{L L} \sum_{i=1}^{3}\left[\left(U_{\mathrm{pmns}}\right)_{e i}\right]^{2} m_{i}\right. \\
& \quad+c_{R R}\left(\frac{M_{W_{L}}^{2}}{M_{W_{R}}^{2}}+\xi\right)^{2} \sum_{i=1}^{3} M_{i} V_{e i}^{* 2} \frac{\mathcal{M}^{0 \nu \beta \beta}\left(M_{i}\right)}{\mathcal{M}^{0 \nu \beta \beta}(0)} \\
& \left.\quad-c_{L R} \theta_{e 1}^{*}\left(\xi+\eta \frac{M_{W_{L}}^{2}}{M_{W_{R}}^{2}}\right)\langle p\rangle\right] \mathcal{M}^{0 \nu \beta \beta}(0) \\
& \equiv m_{\beta \beta} \mathcal{M}^{0 \nu \beta \beta}(0),
\end{aligned}
$$

${ }^{5}$ We refer readers to Ref. [21] for more details. 
where we have made use of Eq. (6) and $c_{L L}, c_{L R}$ and $c_{R R}$ are coefficients which take into account the different chirality of the outgoing electrons. At this point we can make an estimation of the NMEs associated to the heavy neutrino exchange, $\mathcal{M}^{0 \nu \beta \beta}\left(M_{i}\right)$, to understand how relevant the remaining NP contributions are. The effective mass becomes,

$$
\begin{aligned}
\left|m_{\beta \beta}\right|^{2}= & \left|\left(\frac{v_{L}}{v_{R}} M_{R}-\theta M_{R} \theta^{T}\right)_{e e}\right|^{2} \\
& +\left|\left(\xi+\eta \frac{M_{W_{L}}^{2}}{M_{W_{R}}^{2}}\right)\langle p\rangle \theta_{e 1}\right|^{2} \\
& +\left|\left(\frac{M_{W_{L}}^{2}}{M_{W_{R}}^{2}}+\xi\right)^{2}\left\langle p^{2}\right\rangle\left[\left(M_{R}\right)^{-1}\right]_{e e}\right|^{2}
\end{aligned}
$$

where we have neglected the suppressed interference terms between the different chirality contributions [60].

In the rest of this section, we will first study the bounds that can be extracted from the $0 \nu \beta \beta$ decay experiments if one assumes that the three contributions listed in Eq. (27) are completely independent. After that, we will study the region of the parameter space in which a NP signal in the future $0 \nu \beta \beta$ decay experiments can be expected when the correlations among the different contributions are not ignored.

In Fig. 2 we show the constraints on $v_{R}$ (recall that $M_{W_{R}}=$ $g v_{R} / \sqrt{2}$ ) and the mixing between $v_{e L}$ and the lightest heavy neutrino, $\left|\theta_{e}\right|$, extracted from $0 v \beta \beta$ decay experiments when only the contribution from the $W_{L}-W_{R}$ channel (second term in Eq. (27)) is taken into account. In the left panel the mixing $\xi$ saturates the theoretical bound $\left(\xi=M_{W_{L}}^{2} / M_{W_{R}}^{2}\right)$ while in the right panel $\xi$ is neglected. The shaded region is ruled out by the present constraint, $\left|m_{\beta \beta}\right|<0.38 \mathrm{eV}$ [9], while the region

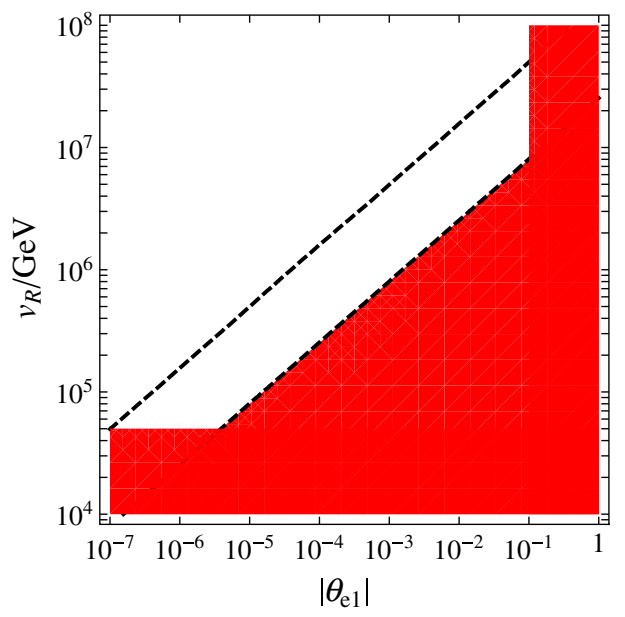

Fig. 2 Heavy regime. The shaded region represents the values of $v_{R}$ and $\left|\theta_{e 1}\right|$ ruled out by the present experimental bound on the $0 v \beta \beta$ decay rate mediated by the $W_{L}-W_{R}$ channel (neglecting the standard and the $W_{R}-W_{R}$ contributions) and the bounds on $M_{W_{R}}$ [40] and non- between the red dashed lines corresponds to the sensitivity of the next-to-next generation of experiments, $10^{-2} \mathrm{eV}<$ $\left|m_{\beta \beta}\right|<0.38 \mathrm{eV}$.

Figure 3 is analogous to Fig. 2, but this time we show the present bound on $v_{R}$ and $\left(Y_{\Delta}\right)_{e e}$ when only the contribution from the $W_{R}-W_{R}$ channel is considered, i.e., only the third term of Eq. (27) is included in the analysis. The future sensitivity is shown as well.

The caveat for Figs. 2 and 3 is that we switch on the NP contributions one at a time without considering the correlation between them and that of the light neutrinos. This is specially problematic if one tries to find the future sensitivity to the parameters of the model. For example, from Fig. 2 one would conclude that $v_{R}$ can be probed in the region $50 \mathrm{TeV} \lesssim v_{R} \lesssim 4 \cdot 10^{4} \mathrm{TeV}$, while from Fig. 3 the conclusion would be different, probing $50 \mathrm{TeV} \lesssim v_{R} \lesssim 500 \mathrm{TeV}$. In this context, the following two questions arise. First, is the standard light neutrino contribution significant for those inputs of the parameters? Can those NP contributions really dominate over the standard one? And second, if yes, for what region of the parameter space? The $0 v \beta \beta$ decay phenomenology is sometimes analyzed taking into account the different contributions one by one, that is, neglecting the rest of the contributions and the correlations induced by the neutrino mass generation mechanism. In this work, we simultaneously include all the relevant contributions in the analysis and emphasize how the correlation plays a vital role in order to answer the previous questions.

In Fig. 4 we show the sensitivity of the next-to-next generation of $0 \nu \beta \beta$ decay experiments $\left(10^{-2} \mathrm{eV}<\left|m_{\beta \beta}\right|<\right.$ $0.38 \mathrm{eV}$ ) to the parameters of the model by including all the relevant contributions and requiring the NP contribution to the $0 v \beta \beta$ decay rate (second and third term in Eq. (27)) to be

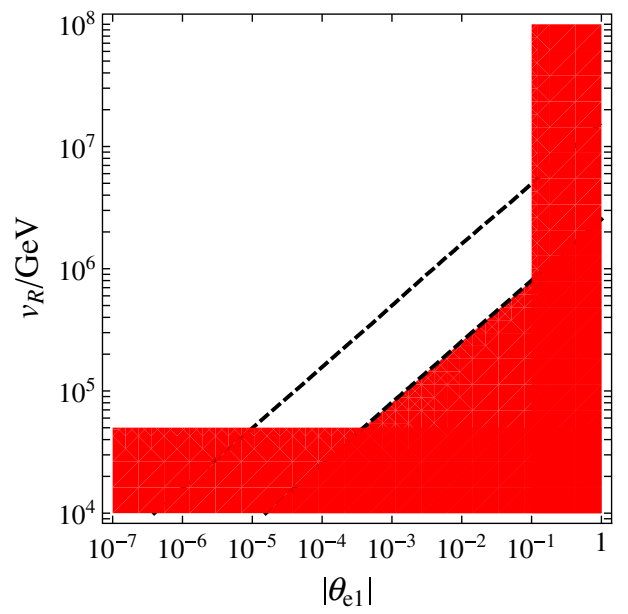

unitarity. The future $0 v \beta \beta$ decay sensitivity, when the standard light neutrino and the $W_{R}-W_{R}$ contributions are not included, is given by the region between the red dashed lines. The mixing $\xi$ has been fixed to $M_{W_{L}}^{2} / M_{W_{R}}^{2}$ (zero) in the left (right) panel 


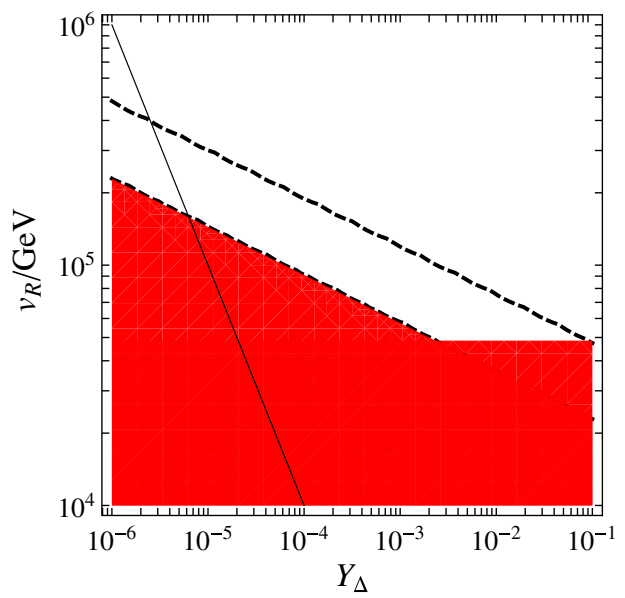

Fig. 3 Heavy regime. The shaded region represents the values of $v_{R}$ and $\left(Y_{\Delta}\right)_{e e}$ ruled out by the present experimental bound on the $0 v \beta \beta$ decay rate mediated by the $W_{R}-W_{R}$ channel (neglecting the standard and the $W_{L}-W_{R}$ contributions) and the bounds on $M_{W_{R}}$ [40]. The future

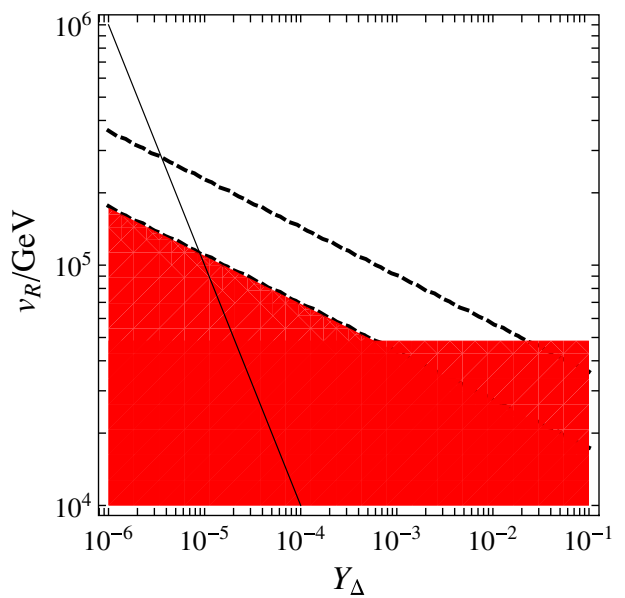

$0 \nu \beta \beta$ decay sensitivity, when the standard light neutrino and the $W_{L^{-}}$ $W_{R}$ contributions are not included, is given by the region between the red dashed lines. The black line corresponds to $\left(M_{R}\right)_{e e}=1 \mathrm{GeV}$. The mixing $\xi$ has been fixed to $M_{W_{L}}^{2} / M_{W_{R}}^{2}$ (zero) in the left (right) panel
Fig. 4 Heavy regime. The shaded region represents the future sensitivity of the next-to-next generation of $0 v \beta \beta$ decay experiments to $v_{R}$ and $\left(Y_{\Delta}\right)_{e e}$ (left panel) and $v_{R}$ and $|\theta|$ (right panel) when the decay rate is dominated by the NP contribution for $\xi=0$ (upper panels) and $\xi=M_{W_{L}}^{2} / M_{W_{R}}^{2}$ (lower panels). In the calculation all the relevant contributions to the process have been included at the same time in the analysis and the present bound on $M_{W_{R}}[40]$ and the active-heavy neutrino mixing $\theta$ is respected. A hierarchical heavy neutrino spectrum has been considered
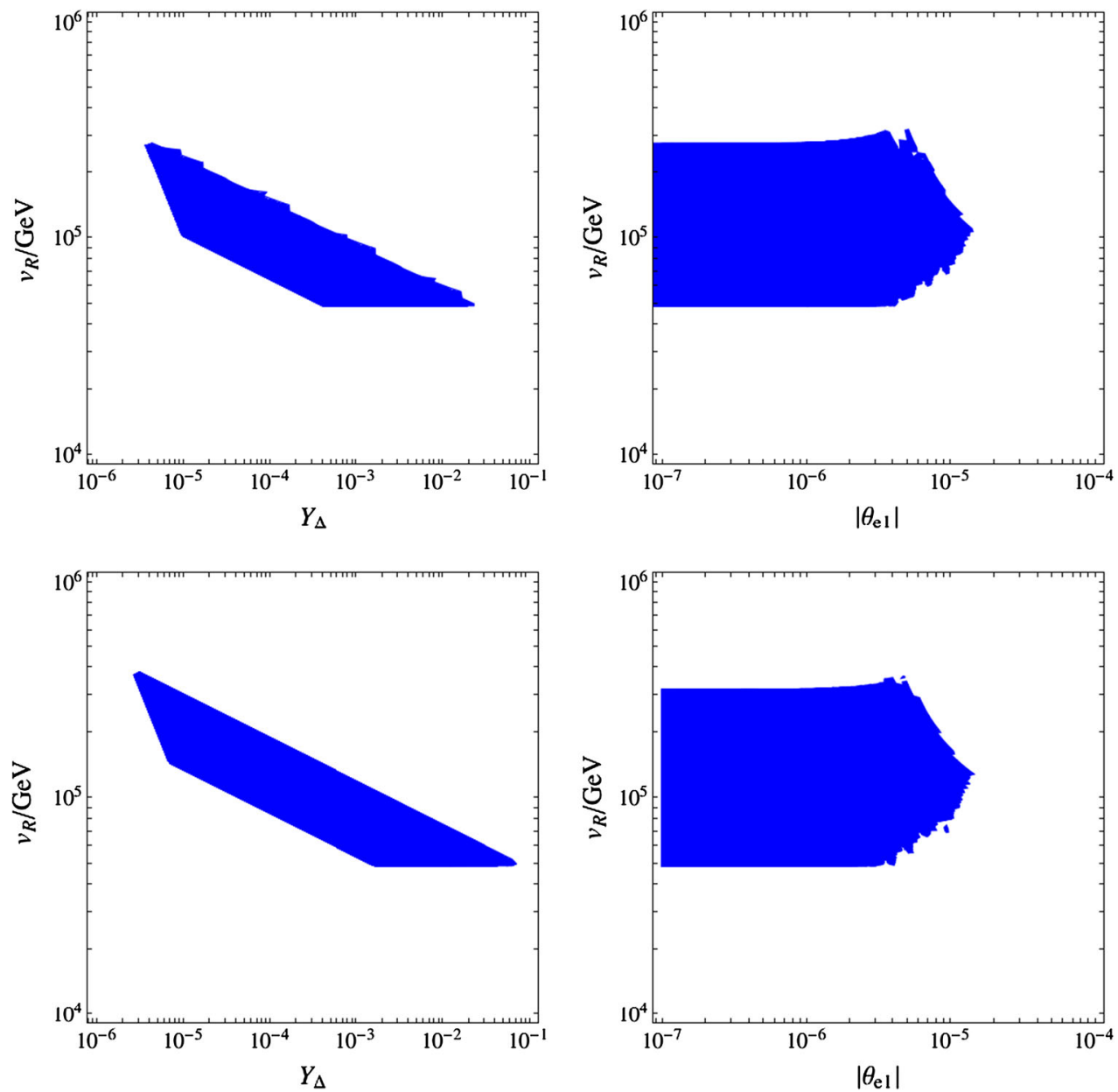

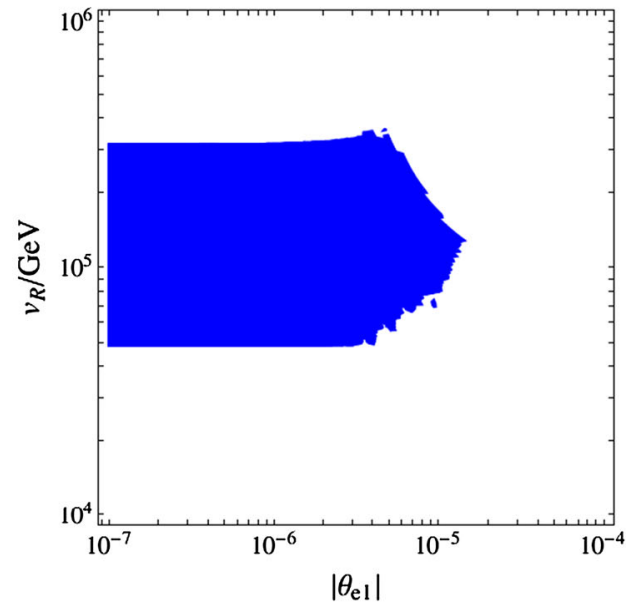

at least 10 times larger than the standard contribution (first term of Eq. (27)). The allowed region is projected onto the $v_{R}-\left(Y_{\Delta}\right)_{e e}$ plane (left panel) and the $v_{R}-\left|\theta_{e 1}\right|$ plane (right panel). The mixing has been neglected in the upper panels while in the lower panels is fixed to its maximum value $\xi=M_{W_{L}}^{2} / M_{W_{R}}^{2}$. The experimental constraints on the $W_{R}$ 
mass [40] and the active-heavy mixing [36,51,52] have also been included. We have assumed that the heavy neutrino spectrum is hierarchical $\left(M_{1} \ll M_{2}, M_{3}\right)$. We confirm that a dominant contribution in the left-right symmetric model coming from NP channels is still possible for the window $50 \mathrm{TeV} \lesssim v_{R} \lesssim 300 \mathrm{TeV}\left(50 \mathrm{TeV} \lesssim v_{R} \lesssim 400 \mathrm{TeV}\right)$ if the trilinear coupling and the mixing are small enough, $3 \cdot 10^{-6} \lesssim\left(Y_{\Delta}\right)_{e e} \lesssim 3 \cdot 10^{-2}\left(3 \cdot 10^{-6} \lesssim\left(Y_{\Delta}\right)_{e e} \lesssim 8 \cdot 10^{-2}\right)$ and $\left|\theta_{e}\right| \lesssim 2 \cdot 10^{-5}$, respectively, for $\xi=0$ (maximal mixing $\xi=M_{W_{L}}^{2} / M_{W_{R}}^{2}$ ). This corresponds to a range of heavy neutrino masses from $\mathrm{GeV}$ to $\mathrm{TeV}$. Comparing the upper and lower panels we can conclude that including the mixing in the analysis has some impact on the results but it is not very significant.

Comparing Fig. 4 with Fig. 2, where only the $W_{L}-W_{R}$ contribution is included, we see that the region of the parameter space which can be experimentally probed shrinks when all the contributions are included at once. From Fig. 2, one could conclude that a NP signal from the $W_{L^{-}} W_{R}$ channel is possible for very large values of $v_{R}$ up to $\sim 10^{4} \mathrm{TeV}$ $\left(M_{W_{R}} \sim 500 \mathrm{TeV}\right)$. However, such a large value of $v_{R}$ requires a quite large mixing $\theta$ since $v_{R}$ suppresses the $W_{L^{-}}$ $W_{R}$ contribution (second term of Eq. (27)) which makes the light neutrino contribution to the $0 v \beta \beta$ decay rate larger than the present bound. Namely, due to the correlation, such a large values of $v_{R}$ and $\theta_{e 1}$ are ruled out and the $W_{L}-W_{R}$ channel cannot give a dominant contribution to the process. On the other hand, even for values of $v_{R}$ close to the present bound, the $W_{L}-W_{R}$ contribution is of the same order of the subleading light-active neutrino one, while the $W_{R}-W_{R}$ contribution becomes larger than that of the $W_{L}-W_{R}$ channel or even above the present experimental bound. Indeed, we have checked numerically that, once the correlations are taken into account, a NP signal can be expected mainly from the $W_{R^{-}}$ $W_{R}$ channel as one can anticipate from the fact that the NP signal regions in Fig. 3 and Fig. 4 (left) overlap. Note that the $W_{L}-W_{R}$ channel could only dominate the decay rate if a cancelation in the light neutrino contribution takes place, a scenario not explored in this work.

\subsection{Light regime}

If the RH neutrinos are lighter than the $0 \nu \beta \beta$ decay scale, $\mathcal{O}(100) \mathrm{MeV}$, the picture significantly changes with respect to the heavy scenario studied above. Equations (16), (19), and (21) remain valid but the NMEs associated with the "heavy" neutrino exchange are not suppressed compared to the light neutrino mediated ones. In fact, for $M_{i}<1 \mathrm{MeV}$, we have in a very good approximation $\mathcal{M}^{0 \nu \beta \beta}(0)=\mathcal{M}^{0 \nu \beta \beta}\left(M_{i}\right)$. This yields a cancelation within the second term of Eqs. (16) and the corresponding amplitude is then given by

$$
A_{L L} \propto\left(M_{L}\right)_{e e} \mathcal{M}^{0 \nu \beta \beta}(0)=\frac{v_{L}}{v_{R}}\left(M_{R}\right)_{e e} \mathcal{M}^{0 \nu \beta \beta}(0),
$$

while Eq. (19) becomes

$A_{R R} \propto\left(\frac{M_{W_{L}}^{2}}{M_{W_{R}}^{2}}+\xi\right)^{2}\left(M_{R}^{*}\right)_{e e} \mathcal{M}^{0 \nu \beta \beta}(0)$,

where we have used Eq. (9). The $W_{L}-W_{R}$ contribution, given by Eq. (21), vanishes due to the unitarity of the $6 \times 6$ neutrino mixing matrix $U$. Therefore, from Eqs. (28)-(29), the expression for the effective mass $m_{\beta \beta}$ when the $\mathrm{RH}$ neutrinos are lighter than $1 \mathrm{MeV}$ becomes

$$
\begin{aligned}
\left|m_{\beta \beta}\right|^{2} & =\left|\frac{v_{L}}{v_{R}}\left(M_{R}\right)_{e e}\right|^{2}+\left|\left(M_{R}^{*}\right)_{e e}\left(\frac{M_{W_{L}}^{2}}{M_{W_{R}}^{2}}+\xi\right)^{2}\right|^{2} \\
& =\left|v_{L}\left(Y_{\Delta}\right)_{e e}\right|^{2}+\left|v_{R}\left(Y_{\Delta}^{*}\right)_{e e}\left(\frac{M_{W_{L}}^{2}}{M_{W_{R}}^{2}}+\xi\right)^{2}\right|^{2} .
\end{aligned}
$$

We conclude that in this regime the $0 \nu \beta \beta$ decay can be completely attributed to the $W_{R}-W_{R}$ channel contribution, if $v_{L} / v_{R} \ll\left(M_{W_{L}} / M_{W_{R}}\right)^{4} \cdot{ }^{6}$

If $v_{L} / v_{R} \gg\left(M_{W_{L}} / M_{W_{R}}\right)^{4}$, the $W_{L}-W_{L}$ channel dominates the decay rate. This does not mean that the standard contribution (that mediated by the light-active neutrinos) always dominates since the RH neutrino exchange can also contribute in this channel. Indeed,

$$
\begin{aligned}
& A_{L L} \propto\left(U_{\mathrm{pmns}} m U_{\mathrm{pmns}}^{T}+\theta M_{R} \theta^{T}\right)_{e e} \mathcal{M}^{0 \nu \beta \beta}(0) \\
& =\frac{v_{L}}{v_{R}}\left(M_{R}\right)_{e e} \mathcal{M}^{0 \nu \beta \beta}(0)
\end{aligned}
$$

Notice that, contrary to the type-I seesaw limit $\left(v_{L} \rightarrow 0\right)$ [24], in this regime $A_{L L}$ does not vanish and the RH neutrinos (second term in the equation above) can contribute to the process. Nevertheless, in this work we will focus on the $W_{R}-W_{R}$ and $W_{L}-W_{R}$ channels. A dominant NP contribution from $W_{L}-W_{L}$ channel mediated by the RH neutrinos will be investigated elsewhere in more detail.

The future sensitivity of the next-to-next generation of $0 \nu \beta \beta$ decay experiments $\left(10^{-2} \mathrm{eV}<\left|m_{\beta \beta}\right|<0.54 \mathrm{eV}\right)$ to the parameters of the model when the $W_{R}-W_{R}$ contribution (second term of Eq. (30)) is at least 10 times larger than that from the $W_{L}-W_{L}$ channel (first term of Eq. (30)) is given in Fig. 5. The allowed region of the parameter space is projected this time onto the $v_{R}-\left(M_{R}\right)_{e e}$ plane (left panel), $v_{R}-\left(Y_{\Delta}\right)_{e e}$ (central panel) and $v_{R}-v_{L}$ (right panel) for $\xi=0$ (solid line) and $\xi=M_{W_{L}}^{2} / M_{W_{R}}^{2}$ (dashed line). The bounds on the $W_{R}$ mass [40] have been also included.

\footnotetext{
${ }^{6}$ Note that the mass of $M_{W_{L}}$ mainly comes from the SM Higgs $\mathrm{VEV}$, not from the $\Delta_{L} \mathrm{VEV}, v_{L}$. As a consequence, $v_{L}$ could be very small. Furthermore, a small $v_{L}$ is in better agreement with the $\rho\left(\equiv M_{W_{L}}^{2} / M_{Z_{L}}^{2} \cos ^{2} \theta_{W}\right)$ parameter constraints.
} 


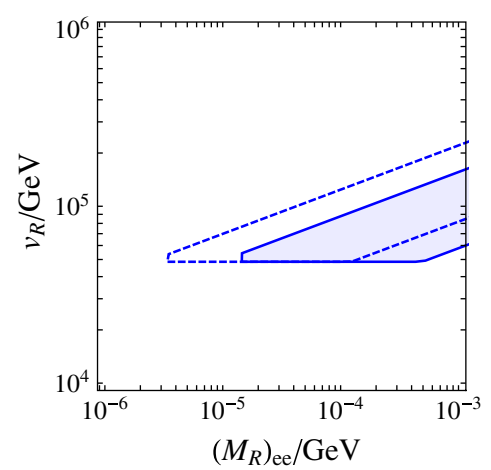

Fig. 5 Light regime. The region inside the solid (dashed) lines represents the future sensitivity of the next-to-next generation of $0 v \beta \beta$ decay experiments when the decay rate is dominated by the $W_{R}-W_{R}$ contribution for $\xi=0\left(\xi=M_{W_{L}}^{2} / M_{W_{R}}^{2}\right)$, projected onto the $v_{R}-\left(M_{R}\right)_{e e}$

Figure 5 (left panel) shows that a NP dominated $0 v \beta \beta$ decay signal can be expected for $\left(M_{R}\right)_{e e} \sim 15 \mathrm{KeV}-1 \mathrm{MeV}$ $\left(\left(M_{R}\right)_{e e} \sim 3 \mathrm{KeV}-1 \mathrm{MeV}\right)$ and $M_{W_{R}} \lesssim 8 \mathrm{TeV}\left(M_{W_{R}} \lesssim\right.$ $12 \mathrm{TeV})$ for $\xi=0\left(\xi=M_{W_{L}}^{2} / M_{W_{R}}^{2}\right)$. This means that in this regime the future sensitivity to $M_{W_{R}}$ is around a factor 2 weaker than in the heavy regime $\left(M_{W_{R}} \lesssim 15-20 \mathrm{TeV}\right.$ depending on the value of $\xi$ ). In both regions the sensitivity is driven by the $W_{R}-W_{R}$ channel. In order to have a dominant $W_{R}-W_{R}$ contribution, the $W_{L}-W_{L}$ one should be of course depleted and this can be achieved for small enough values of $v_{L}\left(v_{L} \lesssim 0.07 \mathrm{GeV}\right)$ as expected from Eq. (30) and shown in Fig. 5 (right panel). One may ask if it is really feasible or natural to have RH neutrinos lighter than $1 \mathrm{MeV}$ while $v_{R}$ is above the $\mathrm{TeV}$. Indeed, this is perfectly possible but requires an uncomfortably small value of the trilinear Yukawa coupling $Y_{\Delta}$ since $M_{i} \sim Y_{\Delta} v_{R}$, as shown in Fig. 5 (left). However, the smallness of $Y_{\Delta}$ could be achieved adding an extra mildly broken global symmetry to the model, as has been done in the popular inverse seesaw models with the lepton number. In such a case these small values of $Y_{\Delta}$ could be considered technically natural since $Y_{\Delta}=0$ would restore the global symmetry. In any case, it should be remarked that a NP signal can only occur for $10^{-10} \lesssim\left(Y_{\Delta}\right)_{e e} \lesssim 10^{-8}$. Finally, comparing the dashed and solid contours we can conclude that the impact of the mixing $\xi$ is not very significant in this region of the parameter space.

\subsection{Mixed scenario}

There is an alternative scenario that has not been studied in the previous sections and consists of the existence of RH neutrinos in both regimes below and above the $0 v \beta \beta$ decay scale. In this section we will focus on the particular case in which one of the RH neutrinos is lighter than $1 \mathrm{MeV}$ and the other two are heavier than $1 \mathrm{GeV}$, i.e., $M_{1}<1 \mathrm{MeV}$ and $M_{2}, M_{3}>1 \mathrm{GeV}$, but the phenomenology remains similar if two RH neutrinos are lighter than $1 \mathrm{MeV}$.

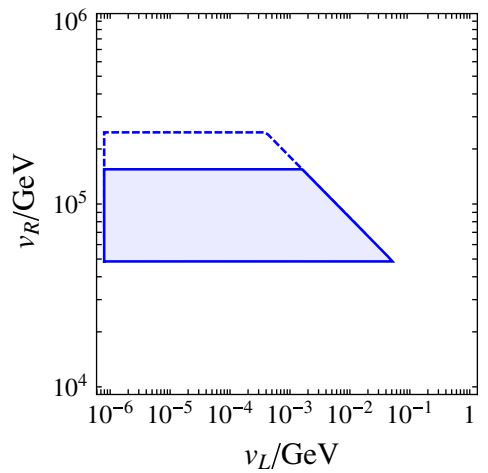

plane (left panel), $v_{R}-\left(Y_{\Delta}\right)_{e e}$ (central panel) and $v_{R}-v_{L}$ (right panel). In the analysis all the relevant contributions have been simultaneously included. The bounds on $M_{W_{R}}$ [40] have been also included

As occurring in the previous section, Eqs. (16), (19), and (21) are also correct in this regime, but only the NMEs associated with the $N_{2}$ and $N_{3}$ exchange are suppressed compared to the light neutrino mediated ones. The NMEs associated with $N_{1}$ satisfy $\mathcal{M}^{0 \nu \beta \beta}(0)=\mathcal{M}^{0 \nu \beta \beta}\left(M_{1}\right)$. As a consequence, in this regime Eqs. (16) and (21) read

$A_{L L} \propto\left[\frac{v_{L}}{v_{R}}\left(V M V^{T}\right)_{e e}-\sum_{i=2}^{3} M_{i}(\theta V)_{e i}^{2}\right] \mathcal{M}^{0 \nu \beta \beta}(0)$,

$A_{L R} \propto-\sum_{i=2}^{3}\left(\theta^{*} V^{*}\right)_{e i} V_{e i}\left(\xi+\eta \frac{M_{W_{L}}^{2}}{M_{W_{R}}^{2}}\right)\langle p\rangle \mathcal{M}^{0 \nu \beta \beta}(0)$,

and Eq. (19) becomes

$$
\begin{gathered}
A_{R R} \propto\left(V_{e 1}^{* 2} M_{1}-\sum_{i=2}^{3} V_{e i}^{* 2} \frac{\langle p\rangle^{2}}{M_{i}}\right) \\
\times\left(\frac{M_{W_{L}}^{2}}{M_{W_{R}}^{2}}+\xi\right)^{2} \mathcal{M}^{0 \nu \beta \beta}(0),
\end{gathered}
$$

where again we have used Eq. (9) and the fact that $\mathcal{M}^{0 \nu \beta \beta}\left(M_{i}\right) / \mathcal{M}^{0 \nu \beta \beta}(0) \ll 1$ for $i=2,3$. Therefore, in this scenario the effective mass $m_{\beta \beta}$ is given by

$$
\begin{aligned}
& \left|m_{\beta \beta}\right|^{2}=\left|\frac{v_{L}}{v_{R}}\left(V M V^{T}\right)_{e e}-\sum_{i=2}^{3} M_{i}(\theta V)_{e i}^{2}\right|^{2} \\
& +\left|\sum_{i=2}^{3}(\theta V)_{e i}^{*} V_{e i}\left(\xi+\eta \frac{M_{W_{L}}^{2}}{M_{W_{R}}^{2}}\right)\langle p\rangle\right|^{2} \\
& +\left|\left(V_{e 1}^{* 2} M_{1}-\sum_{i=2}^{3} V_{e i}^{* 2} \frac{\langle p\rangle^{2}}{M_{i}}\right)\left(\frac{M_{W_{L}}^{2}}{M_{W_{R}}^{2}}+\xi\right)^{2}\right|^{2} .
\end{aligned}
$$



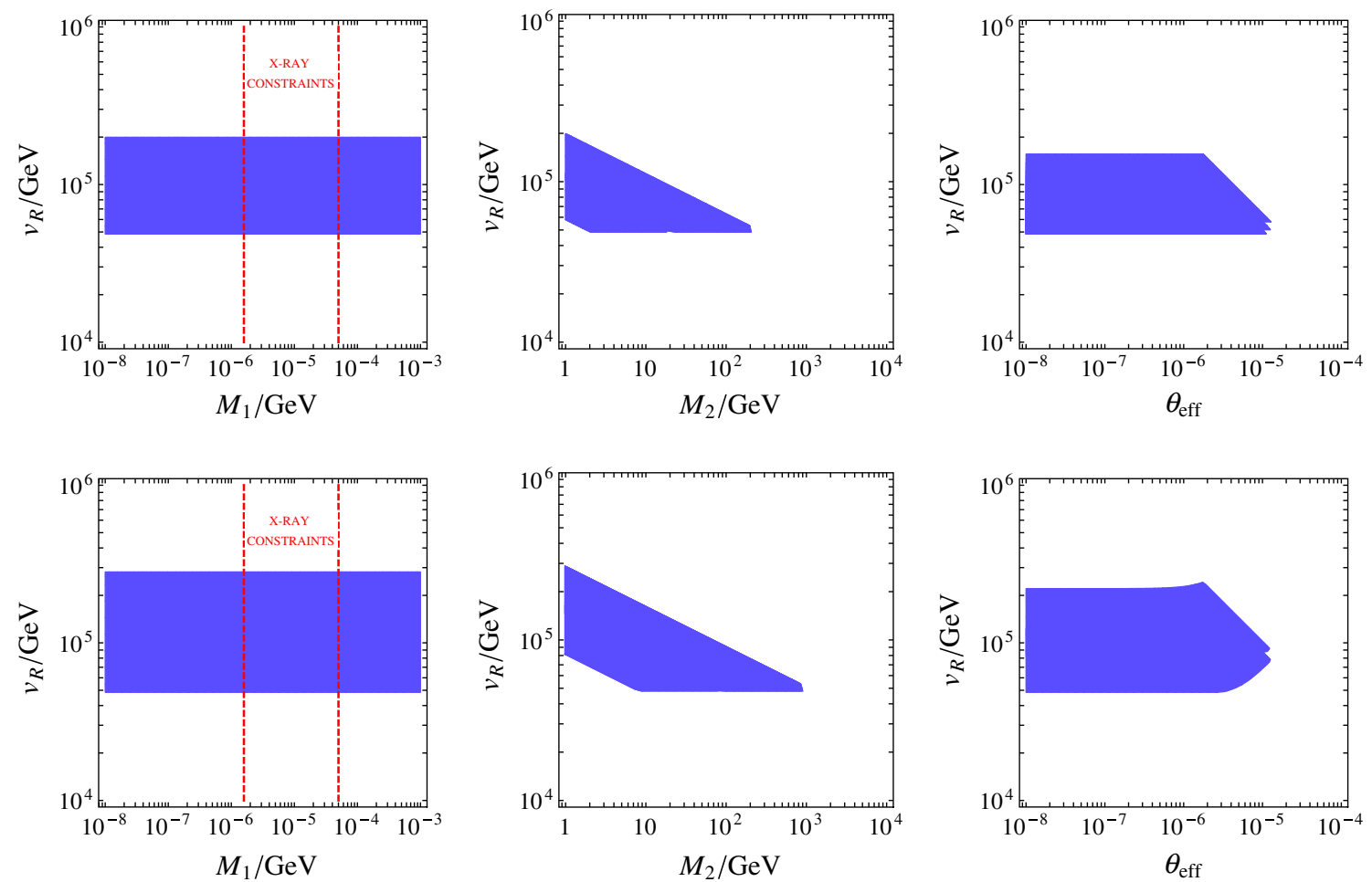

Fig. 6 Mixed scenario. The shaded region represents the future sensitivity of the next-to-next generation of $0 \nu \beta \beta$ decay experiments projected onto the $v_{R}-M_{1}$ plane (left panel), $v_{R}-M_{2}$ (central panel) and $v_{R}-\theta_{\text {eff }}$ (right panel) when the decay rate is dominated by the $W_{L}-W_{R}$ and $W_{R}-W_{R}$ contributions with $\xi=0$ (upper panels) and $\xi=M_{W_{L}}^{2} / M_{W_{R}}^{2}$ (lower panels). In the analysis all the relevant contri-

Contrary to the light regime, in this scenario the $W_{L}-W_{R}$ contribution may be significant. Notice that if $V=I$, the $W_{L}-W_{R}$ contribution cancels out, which means that the $\mathrm{RH}$ neutrino mixing $V$ is very relevant in this region.

In order to be consistent with the rest of this work, associated with the absence of cancelation in the light-active neutrino contribution, we will focus in this section on the limit $\frac{v_{L}}{v_{R}} \rightarrow 0$, neglecting the first term of the $W_{L}-W_{L}$ contribution. As expected, we have checked that if that term is switched on in the analysis, cancelations between the two terms of the $W_{L}-W_{L}$ contribution can perfectly occur for some part of the parameter space leading to better future sensitivities to the $W_{L}-W_{R}$ and $W_{R}-W_{R}$ contributions. On the other hand, we will not study the possibility of having a NP signal from the $W_{L}-W_{L}$ channel mediated by the RH neutrinos since it would also require some level of fine tuning as demonstrated in Ref. [24] for the type-I seesaw case.

The left and central panel of Fig. 6 show the sensitivity of the next-to-next generation of $0 \nu \beta \beta$ decay experiments $\left(10^{-2} \mathrm{eV}<\left|m_{\beta \beta}\right|<0.38 \mathrm{eV}\right)$ to the parameters of the model by including all the relevant contributions and requiring the $W_{L^{-}}-W_{R}$ and $W_{R}-W_{R}$ contribution to the $0 v \beta \beta$ decay rate (second and third term in Eq. (35)) to be at least butions have been simultaneously included. The bounds on $M_{W_{R}}$ [40], the active-"heavy" mixing [52] and the X-ray constraints [61] have been also included. The PMNS angles and oscillation mass-squared differences have been fixed to the central values given in Ref. [62] and $m_{1}=10^{-2} \mathrm{eV}$, while the CP-phases of $U_{\text {pmns }}$ and $V$ have been set to zero

10 times larger than the $W_{L}-W_{L}$ contribution (first term of Eq. (35)) and $\xi=M_{W_{L}}^{2} / M_{W_{R}}^{2}$. The allowed region is projected onto the $v_{R}-M_{1}$ plane (left panel) and $v_{R}-M_{2}$ plane (central panel). We have assumed that $U_{\text {pmns }}$ and $V$ are real and fix the light neutrino mass scale to $m_{1}=10^{-2} \mathrm{eV}$. As in the previous plots, the bounds on the $W_{R}$ mass [40] and the active-"heavy" mixing have been included [36,51,52]. The X-ray constraints [61], which apply if $N_{1}$ is the DM, are also shown in Fig. 5: the region between the red dashed lines is ruled out. We have used Eq. (15) in order to be consistent with the light neutrino mass and mixing pattern as mentioned before. However, in order to illustrate better the impact of the mixing $\theta$, we also show in Fig. 6 (right panel) the sensitivity of the next-to-next generation of $0 \nu \beta \beta$ decay experiments to $v_{R}$ and an effective mixing $\theta_{\text {eff }}$ defined in the following way. We have assumed in Eq. (35) that $\sum_{i=2}^{3}(\theta V)_{e i}^{*} V_{e i} \approx \theta_{\text {eff }}$ and $\sum_{i=2}^{3}(\theta V)_{e i}^{2} M_{i} \approx \theta_{\text {eff }}^{2} M$, which is not true in general but a natural assumption if no particular cancelations are involved. The results for $\xi=0$ are shown in the upper panels while in the lower panels the $W_{L}-W_{R}$ mixing is maximal ( $\xi=M_{W_{L}}^{2} / M_{W_{R}}^{2}$ ). The mixing plays a role only if it is close to the upper bound and even in that case the future sensitivity is similar to the $\xi=0$ limit, as can be observed in Fig. 6. 
Comparing Figs. 5 and 6, it is clear that the sensitivity to $v_{R}$ in this case and the light regime is very similar. The main difference comes from the role of the $W_{L}-W_{R}$ channel, which in the light regime is completely irrelevant but can be significant in the mixed scenario for values of the $W_{L}-W_{R}$ mixing close to the theoretical bound $\xi=M_{W_{L}}^{2} / M_{W_{R}}^{2}$. In fact, saturating the bound, the $W_{L}-W_{R}$ contribution can be the dominant one in the region $\theta_{\text {eff }} \sim 10^{-7}-10^{-5}$ but for smaller values of $\theta_{\text {eff }}$ it is negligible. This is easy to understand since the $W_{L}-W_{R}$ contribution is proportional to $\theta$ while that of the $W_{R}-W_{R}$ channel do not depended on $\theta$. A signal due to the $W_{L}-W_{R}$ channel is possible for such a small values of $\theta$ thanks to the enhancement from $\langle p\rangle$ due to chirality argument and only if $\xi$ is close to its upper bound. On the other hand, the reason why Fig. 6 shows that the $v_{R}$ sensitivity is independent of $M_{1}$ is because for a large part of the parameter space the $W_{R}-W_{R}$ channel dominates, where the $N_{2}$ and $N_{3}$ contribution is important. In summary, a NP $0 v \beta \beta$ decay signal can be expected for a quite small light-sterile neutrino mixing $\left(\theta_{\text {eff }} \lesssim 10^{-5}\right)$ if one of the RH neutrinos is lighter than $1 \mathrm{MeV}$ while the rest are above the $0 \nu \beta \beta$ decay scale. This is interesting regarding the possibility of accommodating the DM in the left-right symmetric models as we will see in the next section.

\section{Complementary constraints}

In this section we will study the impact of the one-loop corrections on the light neutrino masses and whether the part of the parameter space which can be probed in future $0 \nu \beta \beta$ decay experiments, as described above, is accessible by other experiments.

\subsection{One-loop corrections}

Since, in the scenarios studied here, the light neutrino contribution to the $0 \nu \beta \beta$ decay rate is suppressed with respect to the NP ones, one may expect that this significant NP lepton number violation contribution to the $0 \nu \beta \beta$ decay rate could induce non-negligible one-loop corrections to the light neutrino masses. Of course, if the one-loop corrections are larger or similar to the tree-level contribution, they should be included in the analysis, which would modify our previous conclusions. The leading one-loop correction to the light neutrino masses is given by $[63,64]$

$$
\begin{aligned}
\left(\delta M_{L}\right)_{\alpha \beta}= & \frac{1}{(4 \pi v)^{2}}\left(\tilde{m}_{D}^{T}\right)_{\alpha i} \tilde{M}_{i}\left\{\frac{3 \ln \left(\tilde{M}_{i}^{2} / M_{Z}^{2}\right)}{\tilde{M}_{i}^{2} / M_{Z}^{2}-1}\right. \\
& \left.+\frac{\ln \left(\tilde{M}_{i}^{2} / M_{H}^{2}\right)}{\tilde{M}_{i}^{2} / M_{H}^{2}-1}\right\}\left(\tilde{m}_{D}\right)_{i \beta},
\end{aligned}
$$

where $\tilde{m}_{D}$ and $\tilde{M}=\operatorname{diag}\left(M_{1}, M_{2}, M_{3}\right)$ are the Dirac and Majorana sub-matrices, respectively, written in the basis in which the Majorana sub-matrix is diagonal, $M_{Z}$ is the mass of the $Z$ boson and $M_{H}$ the Higgs boson mass. Notice that the self energy diagrams with $W_{L, R}$ bosons in the loop do not give any correction to the light neutrino masses since it is proportional to the external momentum [64]. The contribution would have been sensitive to $\xi$ and $M_{W_{R}}$, had $W_{L, R}$ contributed to the light neutrino corrections. Assuming that there is no fine tuning and the Yukawa couplings are of the same order, we can roughly estimate the size of the one-loop corrections given by Eq. (36) as

$$
\begin{aligned}
& \delta M_{L} / m_{v} \sim 3\left(\frac{M_{z}}{4 \pi v}\right)^{2} \ln \left(M_{i}^{2} / M_{Z}^{2}\right) \\
& +\left(\frac{M_{H}}{4 \pi v}\right)^{2} \ln \left(M_{i}^{2} / M_{H}^{2}\right), \quad \text { for } M_{i} \gg M_{Z}, M_{H}, \\
& \delta M_{L} / m_{v} \sim\left(\frac{M_{i}}{4 \pi v}\right)^{2}\left(3 \ln \left(M_{i}^{2} / M_{Z}^{2}\right)+\ln \left(M_{i}^{2} / M_{H}^{2}\right)\right),
\end{aligned}
$$$$
\text { for } M_{i} \ll M_{Z} \text {. }
$$

Using the estimation given by the first equation above, we can conclude that, if $M_{i} \gg M_{Z}, M_{H}$, the one-loop corrections to the light neutrino masses are under control for the range of values that can be probed in future $0 \nu \beta \beta$ decay experiments. In fact, for $M_{i} \sim 1 \mathrm{TeV}$ we have $\delta M_{L} / m_{v} \sim 10^{-2}$, and $\delta M_{L} / m_{v}$ gets smaller for smaller values of $M_{i}$; for example, $\delta M_{L} / m_{v} \ll 10^{-4}$ for $M_{i} \ll M_{Z}$. This can be understood as follows. From Eq. (36), one can infer that the tree-level contribution is bigger than the loop induced ones because it has a similar structure to $m_{D} M_{i}^{-1} m_{D}^{T}$ but without the loop suppression, $1 /\left(16 \pi^{2}\right)$. This is correct unless some cancelation is at work for the tree-level contribution, which is not the case studied here. Notice that, in this sense, the assumptions made in order to obtain Eq. (37) are quite reasonable.

Therefore, we can conclude that the one-loop corrections to the light neutrino masses are negligible and not relevant in our analysis. The lepton number violation source of the dominant NP contributions studied in the previous sections is the Majorana mass term generated dynamically for the RH neutrinos. Indeed, this source of lepton number violation is related to the light neutrino masses through the seesaw mechanism, and this correlation has been taken into account in the previous analysis. The dominant NP contribution to the $0 \nu \beta \beta$ decay coming from the $W_{R}-W_{R}$ channel (or the $W_{L}-W_{R}$ channel in the mixed scenario) requires the suppression of the standard (and long range) light neutrino one. Since we are not facing the possibility of having any cancelation in the light neutrino contribution, in order to achieve this suppression the Yukawa couplings and $v_{L} / v_{R}$ should be small. The $W_{R}-W_{R}$ contribution can be dominant because the RH mixing is not constrained in contrast with the activeheavy mixing $\theta$, which is necessarily small as the Yukawa 
couplings. The $W_{L}-W_{R}$ channel can dominate in the mixed scenario (only for large $\xi$ ) due to the enhancement coming from the NME and the linear dependence on the active-heavy mixing $\theta$.

\subsection{Other experimental bounds}

The charged LFV experiments are also sensitive to the parameters of the model that can be probed in $0 \nu \beta \beta$ decay experiments. Among them, $\mu \rightarrow e \gamma, \mu \rightarrow 3 e$ and $\mu \rightarrow e$ conversion give the stronger bounds. First of all, the small activeheavy mixing required here in order to have a significant NP contribution to $0 \nu \beta \beta$ decay $\left(\theta \lesssim 10^{-5}\right)$, renders the type-I seesaw like contribution to this processes completely negligible since the strongest present bound coming from $\mu \rightarrow e \gamma$ gives the constraint $\left|\left(\theta^{\dagger} \theta\right)_{e \mu}\right| \lesssim 10^{-5}$. A complete calculation of the charged LFV branching ratios in the MLRSM can be found in [29]. The most relevant constraint in the context of this work comes from $\mu \rightarrow e \gamma$, whose branching ratio, to zeroth order on $\theta$ and $\xi$, is given by

$B r_{\mu \rightarrow e \gamma} \approx 2.6 \times 10^{-10}\left(\frac{\mathrm{TeV}}{M_{W_{R}}}\right)^{4}\left(\frac{\left|\left(M_{R} M_{R}^{*}\right)_{\mu e}\right|}{M_{W_{R}}^{2}}\right)^{2}$,

for $M_{\Delta_{L, R}} \gg M_{W_{R}}$. Applying the present experimental constraint [65] to Eq. (38), the bound on $M_{R}$ reads

$M_{R} \lesssim\left(\frac{M_{W_{R}}^{2}}{4.6 \mathrm{TeV}}\right)$

Saturating the lower bound on $M_{W_{R}}$, one obtains $M_{R} \lesssim$ $1 \mathrm{TeV}$. This is the largest $M_{R}$ that can be probed with $0 \nu \beta \beta$ decay experiments as can be seen in Fig. 4 (it corresponds to the bottom right corner of the shaded regions in the left panels). This means that the future $\mu \rightarrow e \gamma$ experiments can be sensitive at least to that corner of the parameter space, which can also give a signal in $0 \nu \beta \beta$ decay experiments. One should, however, keep in mind that the flavor structure of $M_{R}$ plays an important role, being $\mu \rightarrow e \gamma$ experiments indeed sensitive to $\left(M_{R} M_{R}^{*}\right)_{\mu e}$ and $0 \nu \beta \beta$ decay mainly to $\left(M_{R}\right)_{e e}$ if the heavy neutrino spectrum is hierarchical. Therefore, a NP signal in $0 v \beta \beta$ decay experiments does not necessarily imply also a signal in future $\mu \rightarrow e \gamma$ experiments. On the other hand, the bound in Eq. (39) has been extracted assuming that $M_{\Delta_{L, R}} \gg M_{W_{R}}$, but smaller masses of the triplets can clearly enhance the branching ratio [29]. The same applies for the $\mu \rightarrow 3 e$ and $\mu \rightarrow e$ conversion case since their branching ratios are inversely proportional to the triple masses. Therefore, we cannot extract a bound like Eq. (39) from $\mu \rightarrow 3 e$ and $\mu \rightarrow e$ conversion, since for large triplet masses the branching ratios are very suppressed.
So far, in our LFV analysis we have neglected the $\xi$ contribution. If one switches on the left-right mixing $\xi$, the following constraint from $\mu \rightarrow e \gamma$ can be extracted [33]:

$\left|\left(m_{D}\right)_{\mu e}\right| \xi \lesssim 2 \mathrm{KeV}$

which can be roughly translated into $\theta \xi \lesssim \frac{2 \mathrm{KeV}}{M_{R}}$, which is basically compatible with most of the parameter space that can give a NP signal in $0 \nu \beta \beta$ decay, since $\xi<10^{-3}$ and $\theta<10^{-5}$. Only if $M_{R}$ is close to $\mathrm{TeV}$ and $\xi$ saturates the present bound $\left(\xi \leq M_{W_{L}} / M_{W_{R}}<10^{-3}\right)$, then could the mixing $\xi$ have an impact on $\mu \rightarrow e \gamma$. Basically, we could probe the same part of the parameter space as commented on above but for values of $\xi$ close to its present bound, again with the important warning that the flavor structure plays an essential role here.

Finally, in the MLRSM the electric dipole moment (EDM) can be considerably enhanced with respect to the SM result (up to 10 orders of magnitude). This is because the SM contribution to the EDM appears at four loops while the left-right symmetric model can provide a huge enhancement due to the left-right mixing $\xi$ [66]. In fact, the EDM experiments can be sensitive in the future to part of the parameter space studied here [31], mainly through the imaginary part of $\left[\left(m_{D}\right)_{e e} \xi\right]$.

\section{Dark matter}

In this section we study the possibility of having a successful DM candidate in the context of the MLRSM when the $0 \nu \beta \beta$ decay rate is dominated by NP contributions. The first question which arises from the results of the previous section is whether $N_{1}$ can be DM in the light regime, namely with mass $M_{1} \lesssim \mathcal{O}(\mathrm{MeV})$. This reminds us of the Dodelson-Widrow (DW) scenario [67], where a $\mathrm{KeV}$ neutrino is produced via neutrino oscillations and can be a viable DM candidate. ${ }^{7}$ In the left-right symmetric models, however, a $\mathrm{RH} \mathrm{KeV}$ neutrino $N_{1}$ would be thermally produced via the $W_{R}$ or $Z_{R}$ exchange and decouples from the thermal bath at the freezeout temperature $T_{f}$,

$T_{f} \sim 400 \mathrm{MeV}\left(\frac{g_{*}\left(T_{f}\right)}{70}\right)^{1 / 6}\left(\frac{M_{W_{R}}}{5 \mathrm{TeV}}\right)^{4 / 3}$,

where $g_{*}\left(T_{f}\right)$ is the number of relativistic degrees of freedom at freeze-out. The rule of thumb to estimate $T_{f}$ is to set the interaction rate equal to the expansion rate of the Universe. Given that we are interested on the region of the parameter space in which the NP dominates the $0 v \beta \beta$ decay rate, the $W_{R}$ mass should be in the range $M_{W_{R}} \sim 1-15 \mathrm{TeV}$ (see Fig. 6).

\footnotetext{
7 A recent study for KeV-neutrino DM on the $0 \nu \beta \beta$ decay in the context of the type-I seesaw can be found in Ref. [68], where the KeV-neutrino contribution to the $0 \nu \beta \beta$ rate is subleading due to the $\mathrm{X}$-ray bound.
} 
Therefore, $N_{1}$ is highly relativistic $\left(M_{1} \lesssim \mathrm{MeV} \ll T_{f}\right)$ at freeze-out and the resulting relic density is [69]

$\Omega_{N_{1}} \simeq 3.3\left(\frac{M_{1}}{1 \mathrm{KeV}}\right)\left(\frac{70}{g_{*}\left(T_{f}\right)}\right)$,

which, for $M_{1} \sim \mathrm{KeV}$, would be much larger than the observed DM relic density $\Omega_{\mathrm{DM}}=0.265$ [70]. This constraint is much severer than the $\mathrm{X}$-ray constraints shown in Fig. 5 and the Big Bang Nucleosynthesis (BBN) bound, which is indeed still compatible at $\sim 2 \sigma$ with the existence of one extra relativistic species [71,72]. A possible way out has recently been proposed and studied in detail in Refs. $[69,73]$. Basically, the idea is to dilute the number density of $N_{1}$ by the injection of entropy into the thermal bath after $N_{1}$ freezes out. To be more specific, the out-ofequilibrium decay of $N_{2}$ and/or $N_{3}$, of mass around $\mathrm{GeV}$, into SM particles can increase the entropy of the Universe, leading to faster Universe expansion and in turn a smaller $N_{1}$ density. The set of constraints that should be satisfied if $N_{1}$ as DM was once in thermal equilibrium has been summarized in Ref. [73]. In particular, the authors claim that the required entropy injection can be achieved if $M_{W_{R}} \gtrsim 10$ $16 \mathrm{TeV}$, while the Lyman- $\alpha$ constraints require $M_{1} \gtrsim 1 \mathrm{KeV}$. It turns out that these bounds and the rest of the constraints listed in Ref. [73] are compatible with a future NP signal in $0 v \beta \beta$ decay experiments described in Sect. 3.3. Notice that the RH neutrino spectrum required to have DM $\left(M_{1} \sim \mathrm{KeV}\right.$ and $M_{2}, M_{3} \sim 1-10 \mathrm{GeV}$ ) belongs to the mixed scenario where a NP signal in $0 \nu \beta \beta$ decay experiments is possible. It should be remarked that the constraint $M_{W_{R}} \gtrsim 10-16 \mathrm{TeV}$ is in obvious tension with a future NP signal in the $0 v \beta \beta$ decay. However, as we have mentioned in the previous section, if $v_{L}$ is switched on in the above analysis a cancelation between the two terms in the light neutrino contribution can take place such that a NP signal is possible for $M_{W_{R}} \gtrsim 16 \mathrm{TeV}$.

On the other hand, in Ref. [69] an alternative scenario able to relax the bound on $M_{W_{R}}$ from Ref. [73] was carefully analyzed. In this scenario the desired dilution of the number density of $N_{1}$ is achieved for $M_{1} \simeq 0.5 \mathrm{KeV}, M_{2} \sim 140 \mathrm{MeV}$, and $M_{3} \sim 245 \mathrm{MeV}$, with $M_{W_{R}} \sim 5 \mathrm{TeV}$ and the help of a particular right-handed flavor structure such that $\mathrm{N}_{2}$ 's coupling constant to SM leptons is stronger than that of $N_{1}$. We refer the readers to Ref. [69] for the details of the analysis. In any case, as was already pointed out in Ref. [69], the contribution to the $0 \nu \beta \beta$ decay rate from the $W_{R}-W_{R}$ channel associated with such spectrum can be testable in the future $0 \nu \beta \beta$ decay experiments as we have confirmed in Sect. 3.3. However, we would like to remark that the $W_{L}-W_{R}$ contribution can also be very relevant in this case, as was explained in the previous section.

Finally, in the left-right symmetric models, in principle the neutral component of the right-handed triplet $\Delta_{R}^{0}$, which is a singlet under the SM gauge group, could also be a DM candidate. However, it decays at one-loop into two photons via $W_{R}$ exchange [69], i.e.,

$\Gamma_{\Delta_{R}^{0} \rightarrow \gamma \gamma} \sim 10^{-52} \mathrm{GeV}\left(\frac{m_{\Delta}}{\mathrm{KeV}}\right)^{3}\left(\frac{10^{13} \mathrm{GeV}}{M_{W_{R}}}\right)^{2}$.

The X-ray constraints on $\mathrm{KeV} \mathrm{DM}$ resulting from observations on galaxies and clusters of galaxies [74] requires $\tau_{\Delta_{R}^{0} \rightarrow \gamma \gamma}=1 / \Gamma_{\Delta_{R}^{0} \rightarrow \gamma \gamma} \gtrsim 10^{28} \mathrm{~s}$ or $\Gamma_{\Delta_{R}^{0} \rightarrow \gamma \gamma} \lesssim 10^{-52} \mathrm{GeV}$. Therefore, a KeV $\Delta_{R}^{0}$ would imply a too heavy $W_{R}$ such that the contribution of the NP channels involving $W_{R}$ to the $0 \nu \beta \beta$ decay would be completely negligible. Nevertheless, the X-ray constraints apply only to DM with masses around 1-20 KeV. In principle this leaves another window of $\Delta_{R}^{0}$ mass which can be studied. However, other constraints make this possibility quite unfeasible. First, the mass of DM is constrained to be larger than $\mathrm{KeV}$ [75] because of the Lyman- $\alpha$ observations. Second, for $M_{\Delta_{R}^{0}} \gtrsim 20 \mathrm{KeV}, \tau_{\Delta_{R}^{0}}$ still has to be longer than the age of the Universe, around $10^{18} \mathrm{~s}$, which results again in a very heavy $W_{R}$ that renders any NP contribution to $0 \nu \beta \beta$ decay far beyond the future experimental sensitivity.

In summary, in spite of the existence of various constraints, the left-right symmetric models can accommodate a KeV RH neutrino as a successful DM candidate which can lead to a NP signal in the next-to-next generation of $0 v \beta \beta$ decay experiments.

\section{Conclusions}

We have studied the $0 \nu \beta \beta$ decay phenomenology in the MLRSM. In particular, we have analyzed under which conditions a $0 \nu \beta \beta$ decay signal can come mainly from NP contributions associated with this model. Special attention has been paid to the correlation among the different NP contributions and the standard light neutrino one. This correlation emerges from the neutrino mass generation mechanism and should always be considered in the analysis. The scenario in which an accidental cancelation in the $W_{L}-W_{L}$ contribution takes place has not been explored. The role of the $W_{L}-W_{R}$ mixing $\xi$ and the possibility of having a NP dominated $0 \nu \beta \beta$ decay signal compatible with DM is also investigated.

We have distinguished three different regions of the parameter space based on the mass of the RH neutrinos: (i) all the masses heavier than $\mathrm{GeV}$, denoted by heavy regime; (ii) masses lighter than $\mathrm{MeV}$, dubbed light regime; (iii) the lightest mass below the $\mathrm{MeV}$ and the rest above $\mathrm{GeV}$, called mixed scenario. Notice that (i) has been extensively studied in the literature, but (ii) and (iii) have not been analyzed before in detail for the left-right symmetric models (at least the $0 v \beta \beta$ decay phenomenology). 
In the heavy region, we have found that the dominant NP contribution emerges mainly from the $W_{R}-W_{R}$ channel mediated by the heavy neutrinos. To be more precise, it has been shown that this dominant NP contribution could be measured in the next-to-next generation of $0 \nu \beta \beta$ decay experiments for the window $M_{W_{R}} \sim 1-15 \mathrm{TeV}\left(M_{W_{R}} \sim\right.$ $1-20 \mathrm{TeV})$, if the active-heavy mixing is smaller than $\sim 10^{-5}$ and the right-handed triplet "Yukawa" coupling satisfies $3 \cdot 10^{-6} \lesssim\left(Y_{\Delta}\right)_{e e} \lesssim 3 \cdot 10^{-2}\left(3 \cdot 10^{-6} \lesssim\left(Y_{\Delta}\right)_{e e} \lesssim\right.$ $8 \cdot 10^{-2}$ ), which corresponds to a range of heavy neutrino masses from $\mathrm{GeV}$ to $\mathrm{TeV}$ for $\xi=0\left(\xi=M_{W_{L}}^{2} / M_{W_{R}}^{2}\right)$. We have also shown that neglecting the present correlation between the various contributions, and that with the light neutrinos in particular, can lead to incorrect results. For instance, it is found that the region of the parameter space which can be experimentally probed when only the $W_{L}-W_{R}$ contribution is included in the analysis shrinks considerably if all the contributions are included at once and their correlations are not neglected.

The results for the light region turn out to be similar to those of the heavy region. We have found that a future $0 \nu \beta \beta$ decay NP signal can come only from the $W_{R}-W_{R}$ channel since the $W_{L}-W_{R}$ contribution cancels out in this regime. In particular, we have shown in which part of the parameter space this is possible and we found a similar but weaker sensitivity of the next-to-next generation of $0 \nu \beta \beta$ decay experiments: $M_{W_{R}} \lesssim 8 \mathrm{TeV}\left(M_{W_{R}} \lesssim 12 \mathrm{TeV}\right)$ for $\xi=0\left(\xi=M_{W_{L}}^{2} / M_{W_{R}}^{2}\right)$. A NP signal can be expected for $\left(M_{R}\right)_{e e} \sim 15 \mathrm{KeV}-\mathrm{MeV}\left(\left(M_{R}\right)_{e e} \sim \mathrm{KeV}-\mathrm{MeV}\right)$ for $\xi=0\left(\xi=M_{W_{L}}^{2} / M_{W_{R}}^{2}\right)$, while the triplet Yukawa coupling should be inside the region $10^{-10} \lesssim\left(Y_{\Delta}\right)_{e e} \lesssim 10^{-8}$. This uncomfortably small value of $Y_{\Delta}$ is required in order to have very light $\mathrm{RH}$ neutrinos $\left(M_{i}<1 \mathrm{MeV}\right)$ since $M_{i} \sim Y_{\Delta} v_{R}$; that seems unnatural but might be achieved with the help of an additional global symmetry. On the other hand, notice that in this regime the $W_{L}-W_{L}$ contribution is proportional to $v_{L} / v_{R}$ and therefore a small value of $v_{L}$ $\left(v_{L} \lesssim 0.07 \mathrm{GeV}\right)$ guarantees a dominant $W_{R}-W_{R}$ contribution to the $0 \nu \beta \beta$ decay rate. If $v_{L} / v_{R} \gg\left(M_{W_{L}} / M_{W_{R}}\right)^{4}$, which is still experimentally allowed, the $\mathrm{RH}$ neutrinos can dominate the process via the $W_{L}-W_{L}$ channel, contrary to the type-I seesaw case in which the decay rate is very suppressed if all the RH neutrinos are lighter than the $0 \nu \beta \beta$ decay scale.

In the intermediate regime, with $\mathrm{RH}$ neutrinos in both regions $\left(M_{1} \lesssim 1 \mathrm{MeV}\right.$ and $\left.M_{2}, M_{3} \gtrsim 1 \mathrm{GeV}\right)$, if the $W_{L}-W_{R}$ mixing is close to the theoretical upper bound $\xi=M_{W_{L}}^{2} / M_{W_{R}}^{2}$, the role of the $W_{L}-W_{R}$ channel can be relevant, in contrast with the previous cases. We have found that a NP signal coming from the $W_{L}-W_{R}$ channel could take place for $M_{W_{R}} \sim 1-10 \mathrm{TeV}$ and an active-heavy neutrino mixing $\theta \sim 10^{-7}-10^{-5}$. Indeed, a signal from the $W_{R}-W_{R}$ channel can be expected in a larger region, even for smaller values of $\theta$ since its contribution is independent of the activeheavy neutrino mixing. In this case we have focused our study on the limit $v_{L} / v_{R} \rightarrow 0$, but we have checked that if $v_{L}$ is switched on in the analysis the future sensitivity to $v_{R}$ is much better. However, this can only take place when there is a cancelation between the type-I and type-II seesaw terms in the light neutrino contribution.

In order to study the impact of the $W_{L}-W_{R}$ mixing $\xi$, we have analyzed the following two extreme limits: $\xi=$ $M_{W_{L}}^{2} / M_{W_{R}}^{2}$ (maximal) and $\xi=0$ (negligible). We have shown that the inclusion of the $W_{L}-W_{R}$ mixing $\xi$ can have some impact on the results but it is not very significant, with the possible exception of the mixed scenario where a large mixing is required in order to have a relevant role of the $W_{L}-W_{R}$ channel. In general, due to the enhancement on the $W_{R}-W_{R}$ contribution for maximal mixing, the sensitivity to $M_{W_{R}}$ is about a factor 1.5 larger for $\xi=M_{W_{L}}^{2} / M_{W_{R}}^{2}$ than for $\xi=0$ in all the regions under study.

We have also analyzed the role of the complementary bounds coming from charged LFV processes and the impact of the one-loop corrections to the light neutrino masses in the context of this work. It turns out that the light neutrino masses are stable under one-loop corrections since they might be important only if a cancelation takes places in the light neutrino masses, but not in the case studied here where there is a general suppression of the light masses with small $v_{L} / v_{R}$ and the Yukawa couplings. Future charged LFV experiments might allow us to probe part of the parameter space that can be responsible for a NP signal in $0 \nu \beta \beta$ decay experiments, but only a small region in the heavy regime around $M_{R} \sim 1 \mathrm{TeV}$ (the bottom right corner of the shaded regions in the left panels of Fig. 4). In fact, a more complete study, beyond the scope of this work, including the effect of triplet masses close to their lower bounds, which can enhance the branching ratios, would be required in order to clarify the issue. A large left-right mixing $\xi$ can also be probed in future EDM experiments as was shown in Ref. [31].

Finally, the following DM-related question has also been addressed. Can a NP dominated $0 \nu \beta \beta$ decay signal be compatible with a successful DM candidate in the left-right symmetric models? We conclude that, regardless of the various strong constraints, it is still possible for the scenario proposed in Ref. [73], where a $\mathrm{KeV} \mathrm{RH}$ neutrino can be the DM if the scale of the other heavy neutrinos is around 1-10 GeV and $M_{W_{R}} \gtrsim 10-15 \mathrm{TeV}$. We have shown that the $0 \nu \beta \beta$ decay signal can be induced by the RH neutrinos through the $W_{L}-W_{R}$ and $W_{R}-W_{R}$ channel. Additionally, Ref. [69] opens a window of $M_{W_{R}} \sim 5 \mathrm{GeV}$ within the horizon of LHC after the QCD phase transition is carefully included. 
Acknowledgments We thank to S. Petcov and Javier Menendez for useful discussions and important remarks. This work was partially supported by the ITN INVISIBLES (Marie Curie Actions, PITN- GA2011-289442). W.-C.H. would like to thank the hospitality of IFPA at Université de Liège, where part of this work was performed.

Open Access This article is distributed under the terms of the Creative Commons Attribution License which permits any use, distribution, and reproduction in any medium, provided the original author(s) and the source are credited.

Funded by $\mathrm{SCOAP}^{3}$ / License Version CC BY 4.0.

\section{References}

1. G. Aad et al. [ATLAS Collaboration], Phys. Lett. B 716, 1 (2012). arXiv:1207.7214 [hep-ex]

2. S. Chatrchyan et al. [CMS Collaboration], Phys. Lett. B 716, 30 (2012). arXiv:1207.7235 [hep-ex]

3. P. Minkowski, Phys. Lett. B 67, 421 (1977)

4. M. Gell-Mann, P. Ramond, R. Slansky, Conf. Proc. C 790927, 315 (1979)

5. T. Yanagida, Conf. Proc. C 7902131, 95 (1979)

6. R.N. Mohapatra, G. Senjanovic, Phys. Rev. Lett. 44, 912 (1980)

7. M. Fukugita, T. Yanagida, Phys. Lett. B 174, 45 (1986)

8. C. Arnaboldi et al. [CUORE Collaboration], Nucl. Instrum. Methods A 518, 775 (2004). hep-ex/0212053

9. M. Auger et al. [EXO Collaboration], Phys. Rev. Lett. 109, 032505 (2012). arXiv:1205.5608 [hep-ex]

10. S. Schonert et al. [GERDA Collaboration], Nucl. Phys. Proc. Suppl. 145, 242 (2005)

11. [KamLAND-Zen Collaboration], Phys. Rev. C 85, 045504 (2012). arXiv:1201.4664 [hep-ex]

12. R. Gaitskell et al. [Majorana Collaboration], nucl-ex/0311013

13. J. Diaz, N. Yahlali, M. Ball, J.A.S. Barata, F.I.G.M. Borges, E. Calvo, S. Carcel, J.M. Carmona et al., J. Phys. Conf. Ser. 179, 012005 (2009)

14. A.S. Barabash [NEMO Collaboration], Phys. Atom. Nucl. 67, 1984 (2004)

15. A. Faessler, A. Meroni, S.T. Petcov, F. Simkovic, J. Vergados, Phys. Rev. D 83, 113003 (2011). arXiv:1103.2434 [hep-ph]

16. A. Meroni, S.T. Petcov, F. Simkovic, JHEP 1302, 025 (2013). arXiv: 1212.1331

17. M. Magg, C. Wetterich, Phys. Lett. B 94, 61 (1980)

18. J. Schechter, J.W.F. Valle, Phys. Rev. D 22, 2227 (1980)

19. C. Wetterich, Nucl. Phys. B 187, 343 (1981)

20. G. Lazarides, Q. Shafi, C. Wetterich, Nucl. Phys. B 181, 287 (1981)

21. R.N. Mohapatra, G. Senjanovic, Phys. Rev. D 23, 165 (1981)

22. R. Foot, H. Lew, X.G. He, G.C. Joshi, Z. Phys, C 44, 441 (1989)

23. J. Lopez-Pavon, S. Pascoli, C.-F. Wong, Phys. Rev. D 87, 093007 (2013). arXiv:1209.5342 [hep-ph]

24. M. Blennow, E. Fernandez-Martinez, J. Lopez-Pavon, J. Menendez, JHEP 1007, 096 (2010). arXiv: 1005.3240 [hep-ph]

25. J.C. Pati, A. Salam, Phys. Rev. D 10, 275 (1974) [Erratum-ibid. D 11, 703 (1975)]

26. R.N. Mohapatra, J.C. Pati, Phys. Rev. D 11, 2558 (1975)

27. G. Senjanovic, R.N. Mohapatra, Phys. Rev. D 12, 1502 (1975)

28. M. Nemevsek, F. Nesti, G. Senjanovic, V. Tello, arXiv:1112.3061 [hep-ph]

29. V. Tello, Connections between the high and low energy violation of lepton and flavor numbers in the minimal left-right symmetric model. PhD thesis, SISSA, Sept 2012. http://www.sissa.it/tpp/ phdsection/AlumniThesis/Vladimir\%20Tello
30. J. Chakrabortty, H.Z. Devi, S. Goswami, S. Patra, JHEP 1208, 008 (2012). arXiv:1204.2527 [hep-ph]

31. M. Nemevsek, G. Senjanovic, V. Tello, Phys. Rev. Lett. 110(15), 151802 (2013). arXiv:1211.2837 [hep-ph]

32. M.K. Parida, S. Patra, Phys. Lett. B 718, 1407 (2013). arXiv: 1211.5000 [hep-ph]

33. J. Barry, W. Rodejohann, JHEP 1309, 153 (2013). arXiv: 1303.6324 [hep-ph]

34. P.S. Bhupal Dev, S. Goswami, M. Mitra, W. Rodejohann, Phys. Rev. D 88, 091301 (2013). arXiv:1305.0056 [hep-ph]

35. C. -H. Lee, P. S. Bhupal Dev, R. N. Mohapatra, Phys. Rev. D 88, 093010 (2013). arXiv:1309.0774 [hep-ph]

36. S. Antusch, C. Biggio, E. Fernandez-Martinez, M.B. Gavela, J. Lopez-Pavon, JHEP 0610, 084 (2006). hep-ph/0607020

37. A. Melfo, M. Nemevsek, F. Nesti, G. Senjanovic, Y. Zhang, Phys. Rev. D 85, 055018 (2012). arXiv:1108.4416 [hep-ph]

38. J. Beringer et al. [Particle Data Group Collaboration], Phys. Rev. D 86, 010001 (2012)

39. V.M. Abazov et al. [D0 Collaboration], Phys. Lett. B 699, 145 (2011). arXiv:1101.0806 [hep-ex]

40. G. Aad et al. [ATLAS Collaboration], Phys. Rev. Lett. 109, 081801 (2012). arXiv:1205.1016 [hep-ex]

41. J.A. Casas, A. Ibarra, Nucl. Phys. B 618, 171 (2001). hep-ph/0103065

42. E.K. Akhmedov, W. Rodejohann, JHEP 0806, 106 (2008). arXiv:0803.2417 [hep-ph]

43. A. Ibarra, E. Molinaro, S.T. Petcov, Phys. Rev. D 84, 013005 (2011). arXiv:1103.6217 [hep-ph]

44. M. Mitra, G. Senjanovic, F. Vissani, Nucl. Phys. B 856, 26 (2012). arXiv:1108.0004 [hep-ph]

45. R.N. Mohapatra, J.W.F. Valle, Phys. Rev. D 34, 1642 (1986)

46. G.C. Branco, W. Grimus, L. Lavoura, Nucl. Phys. B 312, 492 (1989)

47. M. Malinsky, J.C. Romao, J.W.F. Valle, Phys. Rev. Lett. 95, 161801 (2005). hep-ph/0506296

48. M. Shaposhnikov, Nucl. Phys. B 763, 49 (2007). hep-ph/0605047

49. M.B. Gavela, T. Hambye, D. Hernandez, P. Hernandez, JHEP 0909, 038 (2009). arXiv:0906.1461 [hep-ph]

50. J.D. Vergados, Phys. Rept. 133, 1 (1986)

51. E. Fernandez-Martinez, M.B. Gavela, J. Lopez-Pavon, O. Yasuda, Phys. Lett. B 649, 427 (2007). hep-ph/0703098

52. A. Atre, T. Han, S. Pascoli, B. Zhang, JHEP 0905, 030 (2009). arXiv:0901.3589 [hep-ph]

53. M. Doi, T. Kotani, E. Takasugi, Prog. Theor. Phys. Suppl. 83, 1 (1985)

54. K. Muto, E. Bender, H.V. Klapdor, Z. Phys. A 334, 187 (1989)

55. E. Caurier, F. Nowacki, A. Poves, J. Retamosa, Phys. Rev. Lett. 77, 1954 (1996)

56. M. Hirsch, H.V. Klapdor-Kleingrothaus, O. Panella, Phys. Lett. B 374, 7 (1996). hep-ph/9602306

57. G. Pantis, F. Simkovic, J.D. Vergados, A. Faessler, Phys. Rev. C 53, 695 (1996). nucl-th/9612036

58. J. Suhonen, O. Civitarese, Phys. Rept. 300, 123 (1998)

59. G. Aad et al. [ATLAS Collaboration], Eur. Phys. J. C 72, 2244 (2012). arXiv:1210.5070 [hep-ex]

60. A. Halprin, S.T. Petcov, S.P. Rosen, Phys. Lett. B 125, 335 (1983)

61. C.R. Watson, J.F. Beacom, H. Yuksel, T.P. Walker, Phys. Rev. D 74, 033009 (2006). astro-ph/0605424

62. M.C. Gonzalez-Garcia, M. Maltoni, J. Salvado, T. Schwetz, JHEP 1212, 123 (2012). arXiv:1209.3023 [hep-ph]

63. A. Pilaftsis, Z. Phys, C 55, 275 (1992). hep-ph/9901206

64. W. Grimus, L. Lavoura, Phys. Lett. B 546, 86 (2002). arXiv:hep-ph/0207229

65. J. Adam et al. [MEG Collaboration], Phys. Rev. Lett. 110, 201801 (2013). arXiv:1303.0754 [hep-ex]

66. J.F. Nieves, D. Chang, P.B. Pal, Phys. Rev. D 33, 3324 (1986) 
67. S. Dodelson, L.M. Widrow, Phys. Rev. Lett. 72, 17 (1994). hep-ph/9303287

68. A. Merle, V. Niro, Phys. Rev. D 88, 113004 (2013). arXiv:1302.2032 [hep-ph]

69. M. Nemevsek, G. Senjanovic, Y. Zhang, JCAP 1207, 006 (2012). arXiv:1205.0844 [hep-ph]

70. P.A.R. Ade et al. [Planck Collaboration], arXiv:1303.5076 [astroph.CO]

71. G. Mangano, P.D. Serpico, Phys. Lett. B 701, 296 (2011). arXiv:1103.1261 [astro-ph.CO]

72. J. Hamann, S. Hannestad, G.G. Raffelt, Y.Y.Y. Wong, JCAP 1109, 034 (2011). arXiv:1108.4136 [astro-ph.CO]
73. F. Bezrukov, H. Hettmansperger, M. Lindner, Phys. Rev. D 81, 085032 (2010). arXiv:0912.4415 [hep-ph]

74. K. Abazajian, G.M. Fuller, W.H. Tucker, Astrophys. J. 562, 593 (2001). astro-ph/0106002

75. M. Viel, G.D. Becker, J.S. Bolton, M.G. Haehnelt, M. Rauch, W.L.W. Sargent, Phys. Rev. Lett. 100, 041304 (2008). arXiv:0709.0131 [astro-ph] 